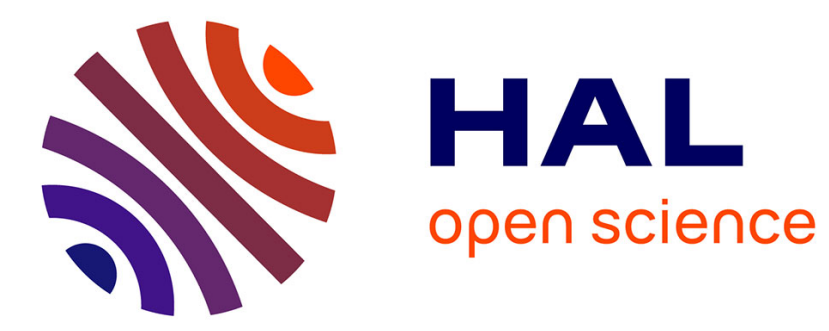

\title{
Counterparty risk and funding: immersion and beyond
}

Stéphane Crépey, Shiqi Song

\section{To cite this version:}

Stéphane Crépey, Shiqi Song. Counterparty risk and funding: immersion and beyond. 2018. hal01764403

\section{HAL Id: hal-01764403 \\ https://hal.science/hal-01764403}

Preprint submitted on 11 Apr 2018

HAL is a multi-disciplinary open access archive for the deposit and dissemination of scientific research documents, whether they are published or not. The documents may come from teaching and research institutions in France or abroad, or from public or private research centers.
L'archive ouverte pluridisciplinaire HAL, est destinée au dépôt et à la diffusion de documents scientifiques de niveau recherche, publiés ou non, émanant des établissements d'enseignement et de recherche français ou étrangers, des laboratoires publics ou privés. 


\title{
Counterparty risk and funding: immersion and beyond
}

\author{
Stéphane Crépey • Shiqi Song *
}

Received: 28 April 2014 / Accepted: 30 March 2016

\begin{abstract}
In Crépey [9], a basic reduced-form counterparty risk modelling approach was introduced under a standard immersion hypothesis between a reference filtration and the filtration progressively enlarged by the default times of the two parties. This basic setup, with a related continuity assumption on some of the data at the first default time of the two parties, is too restrictive for wrong-way and gap risk applications, such as counterparty risk on credit derivatives. This paper introduces an extension of the basic approach, implements it through marked default times and applies it to counterparty risk on credit derivatives.
\end{abstract}

Keywords: Counterparty risk, Funding, BSDE, Reduced-form credit modelling, Immersion, Wrong-way risk, Gap risk, Collateral, Credit derivatives, Marked default times, Marshall-Olkin copula

Mathematics Subject Classification: 91G40, 60H10, $60 \mathrm{G} 07$.

JEL Classification D52 - D53 - G12

\section{Introduction}

Counterparty risk is the risk of default of a counterparty in OTC derivative transactions, a topical issue since the global financial crisis. The default risk of a bank also induces a spread between its unsecured borrowing rate and its investing rate. Hence, banks need

* This research benefited from the support of the "Chair Markets in Transition" under the aegis of Louis Bachelier laboratory, a joint initiative of École polytechnique, Université d'Évry Val d'Essonne and Fédération Bancaire Française.

S. Crépey (corresponding author)

Laboratoire de Mathématiques et Modélisation d'Évry, 91037 Évry Cedex, France

E-mail: stephane.crepey@univ-evry.fr

S. Song

Laboratoire de Mathématiques et Modélisation d'Évry, 91037 Évry Cedex, France 
to compute a total valuation adjustment (TVA) in order to account for counterparty and funding risk.

As is well known since the seminal papers by Korn [23], Cvitanic and Karatzas [14] or El Karoui et al. [16], in presence of different borrowing and lending rates, pricing rules become nonlinear. Accordingly, the TVA equation is a Lipschitz BSDE (see Crépey [9], Brigo and Pallavicini [7] or Bichuch et al. [2]). Moreover, it is posed over a random time interval and may involve a nonstandard, implicit terminal condition at the first default time of a party.

To deal with such equations, a first reduced-form counterparty risk modelling approach has been introduced in Crépey [9], in a basic immersion setup between the reference filtration of the underlying market exposure and the full model filtration progressively enlarged by the default times of the two parties.

This basic immersion setup, with a related continuity assumption on some of the data at the first default time of the two parties, is too restrictive for applications such as counterparty risk on credit derivatives, characterized by wrong-way risk, i.e., adverse dependence between the exposure and the credit risk of the counterparties, and gap risk, i.e., slippage between the portfolio and its collateral during the so-called cure period that separates the default from the liquidation. In the case of credit derivatives one also faces specific dependence and dimensionality challenges.

To tackle these issues, this paper introduces an extended counterparty risk reducedform modelling approach, implements it with marked default times and applies it to counterparty risk on credit derivatives.

The outline of the paper is as follows. In Sect. 2 we present the bilateral counterparty risk and funding setup. In Sect. 3 we derive the full TVA BSDE. Sect. 4 deals with a reduced BSDE that is applicable whenever the first default time of the two parties satisfies the condition (C) in this paper. In Sect. 5 we establish the wellposedness of the TVA BSDEs. In the marked default times framework of Sect. 6 , we derive a CVA/DVA (credit/debt valuation adjustment) and FVA (funding valuation adjustment) decomposition of the TVA. Sect. 7 uses this approach for designing a dynamic Marshall-Olkin (DMO) model of TVA on credit derivatives. A suitable TVA numerical scheme is presented in Sect.8 Numerical results are commented in Sect.9

The main theoretical contribution of this work is to establish the mathematical well-posedness of the full and reduced TVA BSDEs, under a relaxed dependence assumption between the counterparties first-to-default time $\tau$ and the market reference filtration $\mathbb{F}$. From a practical point of view, these results allow modelling a TVA process as a solution to the "simple", reduced TVA BSDE, including in wrong-way and gap risk setups such as the DMO model that we propose for dealing with TVA on credit derivatives. 
Part I

\section{TVA BSDEs}

Throughout the paper we consider a netted portfolio of OTC derivatives, with final maturity $T$, traded between two defaultable counterparties, which we generically refer to as the "contract between the bank and its counterparty". We adopt the perspective of the bank. In particular, a cashflow of \pm 1 means \pm 1 to the bank.

Here is a summary list of notations introduced in the first part of the paper.

CVA, DVA, FVA, TVA Credit valuation adjustment, debt valuation adjustment, funding valuation adjustment, total valuation adjustment.

$\mathbb{G}, \mathbb{F}$ Full model filtration (including the information related to the default of the two counterparties), market reference filtration.

$\mathbb{E}, \widetilde{\mathbb{E}}$ Expectations under the probability measures $\mathbb{Q}$ and $\mathbb{P}$.

$R_{c}, R_{b}, \Lambda \in[0,1]$ Recovery rate of the counterparty to the bank, of the bank to the counterparty, fractional loss of the funder in case of default of the bank.

$T, \delta$ Maturity of the contract, length of the cure (or liquidation) period.

$\tau_{c}, \tau_{b}, \tau, \bar{\tau}, \tau^{\delta}, \bar{\tau}^{\delta}$ Default time of the counterparty, default time of the bank, first default time of the two parties, $\tau \wedge T, \tau+\delta$, time horizon $\bar{\tau}^{\delta}=\mathbb{1}_{\{\tau<T\}} \tau^{\delta}+\mathbb{1}_{\{\tau \geq T\}} T$ of the TVA problem.

$J=\mathbb{1}_{\llbracket 0, \tau \llbracket}, S$ Joint survival indicator process of the bank and the counterparty, Azéma supermartingale of $\tau$

$\tau^{\star}=\tau_{b} \wedge \tau_{c}^{\delta}, \bar{\tau}^{\star}=\mathbb{1}_{\{\tau<T\}} \tau^{\star}+\mathbb{1}_{\{\tau \geq T\}} T, J^{\star}=\mathbb{1}_{\llbracket 0, \tau^{\star} \llbracket}$

$\gamma^{c}, \gamma^{b}, \gamma$ Intensities of $\tau_{c}, \tau_{b}$ and $\tau$.

$U^{\prime} \mathbb{F}$ predictable reduction of a $\mathbb{G}$ predictable process $U$

$r, r+c, r+\lambda, r+\bar{\lambda}, \widetilde{\lambda}=\bar{\lambda}-\gamma^{b} \Lambda$ Risk-free rate, rate of remuneration of the posted collateral, investing rate of the bank, unsecured funding rate of the bank, liquidity funding spread of the bank.

$P$ Risk-free price or mark-to-market of the contract, ignoring counterparty risk and assuming a risk-free funding rate.

$\Delta=\int_{\llbracket 0, \tau^{\star} \rrbracket} e^{\int_{s}^{3} r_{u} d u} d D_{u}$ Cumulative contractual dividends that fail to be paid from time $\tau$ onwards, capitalized at the risk-free rate.

$Q=P+\Delta$ Algebraic debt of the counterparty to the bank (before consideration of the collateral).

$\Pi, \Theta=Q-\Pi$ Risky price of the contract accounting for counterparty and funding risk (as opposed to the risk-free price $P$ ), TVA process.

$V, I^{c} \geq 0, I^{b} \leq 0, C^{c}=V+I^{c}, C^{b}=V+I^{b}, C=V+I^{c}+I^{b}$ Variation margin counted positively when posted by the counterparty and negatively when posted by the bank, initial margin posted by the counterparty, negative of the initial margin posted by the bank, total collateral guarantee for the bank, negative of the total collateral guarantee for the counterparty, negative of the collateral funded by the bank.

$\varepsilon_{c}=\left(Q_{\tau^{\delta}}-C_{\tau}^{c}\right)^{+}, \varepsilon_{b}=\left(Q_{\tau^{\delta}}-C_{\tau}^{b}\right)^{-}$Liquidation debts of the counterparty to the bank, of the bank to the counterparty 
$\chi, \xi=Q_{\tau^{\delta}}-\chi=\mathbb{1}_{\left\{\tau_{c} \leq \tau_{b}^{\delta}\right\}}\left(1-R_{c}\right) \varepsilon_{c}-\mathbb{1}_{\left\{\tau_{b} \leq \tau_{c}^{\delta}\right\}}\left(1-R_{b}\right) \varepsilon_{b}$ Close-out cashflow of the bank, counterparty risk exposure of the bank.

$\mathcal{W},\left(-\mathcal{W}_{\tau_{b}-}-C_{\tau_{b}-}\right)^{+}$Value process of the hedging, collateralization and funding portfolio of the bank, debt of the bank to its funder right before $\tau_{b}$.

$g_{t}(\vartheta)$ Funding coefficient such that $\left(-r_{t} \mathcal{W}_{t}+g_{t}\left(-\mathcal{W}_{t}\right)\right) d t$ represents the bank funding cost over $(t, t+d t)$.

$f_{t}(\vartheta)=g_{t}\left(Q_{t}-\vartheta\right)-r_{t} \vartheta, \widetilde{f}_{t}(\vartheta)$ Coefficients of the full and reduced BSDEs.

$\tau_{e}, \gamma^{e} ; E ; E_{b}, E_{c}$ Stopping time with mark $e$ and intensity $\gamma^{e}$; finite set of marks such that $\tau=\min _{e \in E} \tau_{e}$; subsets of $E$ such that $\tau_{b}=\min _{e \in E_{b}} \tau_{e}, \tau_{c}=\min _{e \in E_{c}} \tau_{e}$.

We write $x^{ \pm}=\max ( \pm x, 0), \int_{a}^{b}=\int_{(a, b]}$. We denote by $\mathcal{B}(\mathbb{S})$ the Borel $\sigma$ field on a topological space $\mathbb{S}$ and by $\mathcal{P}(\mathbb{F}), \mathcal{O}(\mathbb{F})$ and $\mathcal{R}(\mathbb{F})$ the predictable, optional and progressive $\sigma$ fields with respect to a filtration $\mathbb{F}$. Order relationships between random variables (resp. processes) are meant almost surely (resp. in the indistinguishable sense). All time intervals are random unless stated otherwise.

\section{Bilateral counterparty risk and funding setup}

Let $(\Omega, \mathbb{G}, \mathbb{Q})$ represent a risk-neutral pricing stochastic basis, such that all our processes are $\mathbb{G}$ adapted and all the random times of interest are $\mathbb{G}$ stopping times. We suppose that the model filtration $\mathbb{G}=\left(\mathcal{G}_{t}\right)_{t \in \mathbb{R}_{+}}$satisfies the usual conditions and we denote by $\mathbb{E}$ the expectation under $\mathbb{Q}$.

We define the risk-free price $P$ of the contract as its mark-to-market ignoring counterparty risk and assuming a risk-free funding rate, i.e.,

$$
\beta_{t} P_{t}=\mathbb{E}\left(\int_{t}^{T} \beta_{s} d D_{s} \mid \mathcal{G}_{t}\right), t \in[0, T] .
$$

Here the finite variation process $D$ represents the cumulative promised dividend process of the contract (contractual cashflows ignoring counterparty risk); $\beta_{t}=e^{-\int_{0}^{t} r_{s} d s}$ is the risk-neutral discount factor at the risk-free rate $r$, assumed progressively measurable and Lebesgue integrable.

But the two parties in the contract are defaultable. Let $\tau_{b}$ and $\tau_{c}$ stand for the default times of the bank and of the counterparty, modelled as $\mathbb{G}$ stopping times with $\left(\mathbb{G}, \mathbb{Q}\right.$ ) (predictable) intensities $\gamma^{b}$ and $\gamma^{c}$. As a consequence, the first default time of the two parties, $\tau=\tau_{b} \wedge \tau_{c}$, is a stopping time with intensity $\gamma$ such that $\max \left(\gamma^{b}, \gamma^{c}\right) \leq \gamma \leq \gamma^{b}+\gamma^{c}$ (with indistinguishable equality in the right-hand side iff $\tau_{b} \neq \tau_{c}$ holds almost surely). Note that in such an intensity setup, any event $\left\{\tau_{b}=t\right\}$ or $\left\{\tau_{c}=t\right\}$, for any fixed time $t$, has zero probability and can be ignored in the analysis.

An additional feature is a time $\operatorname{lag} \delta \geq 0$, called the cure period, a few days usually, between the first default time $\tau$ of the two parties and the liquidation of the contract (or portfolio). We write

$$
\begin{aligned}
& \bar{\tau}=\tau \wedge T, \quad \tau^{\delta}=\tau+\delta, \quad \bar{\tau}=\mathbb{1}_{\{\tau<T\}} \tau^{\delta}+\mathbb{1}_{\{\tau \geq T\}} T \\
& \tau^{\star}=\tau_{b} \wedge \tau_{c}^{\delta}, \quad \bar{\tau}^{\star}=\mathbb{1}_{\{\tau<T\}} \tau^{\star}+\mathbb{1}_{\{\tau \geq T\}} T .
\end{aligned}
$$


We assume that the bank, having bought the contract from its counterparty at time 0, sets-up a collateralization, hedging and funding portfolio. Collateral consists of cash or various possible eligible securities posted through margin calls as default guarantee by the two parties. If $\tau<T$, then the contractual dividends $d D_{t}$ cease to be paid and the collateral is frozen from time $\tau$ onwards; A close-out cashflow $\chi$ paid to the bank at time $\tau^{\delta}$ closes out its position. Hence, the liquidation procedure results in an effective time horizon $\bar{\tau}^{\delta}$ of the pricing problem. The collateralization, hedging and funding portfolio of the bank is supposed to be held by the bank itself before $\bar{\tau}$ 太 and, if $\tau<T$ and $\tau_{b} \leq \tau_{c}^{\delta}$ (hence $\tau^{\star}=\tau_{b}$ ), taken over by a risk-free liquidator on $\llbracket \bar{\tau}^{\star}, \bar{\tau}^{\delta} \rrbracket$.

Regarding hedging, for simplicity, we restrict ourselves to a securely funded hedge, entirely implemented by means of swaps, short sales or repurchase agreements, at no upfront payment. This assumption encompasses the vast majority of hedges that are used in practice. The meaning of a risk-neutral pricing measure in our setup, with different funding rates in particular, is specified by a martingale condition that will be introduced in the form of the risky price BSDE (3.1). But, in the first place, a pricing measure must be such that the gains associated with the trading of unit positions in the hedging assets, gain processes denoted in vector form by $\mathcal{M}$, are local martingales. This rules out arbitrage opportunities in the market of hedging instruments (provided one restricts attention to hedging strategies resulting in a wealth process bounded from below; see Bielecki and Rutkowski [5, Corollary 3.1] for a formal statement).

Until $\bar{\tau}^{\star}$, the bank needs to fund its position, i.e., the contract and its collateral (the cost of funding the hedge is already accounted for in the hedging assets gain martingale $\mathcal{M})$. We denote by $g=g_{t}(\pi)$ an $\mathcal{R}(\mathbb{G}) \otimes \mathcal{B}(\mathbb{R})$-measurable funding coefficient such that

$$
\left(-r_{t} \mathcal{W}_{t}+g_{t}\left(-\mathcal{W}_{t}\right)\right) d t
$$

represents the bank's funding cost over $(t, t+d t)$, where $\mathcal{W}$ is the value process of the hedging, collateralization and funding portfolio of the bank. In addition, the bank may receive a funding windfall benefit at its own default, modelled as a cashflow

$$
\left(-\mathcal{W}_{\tau_{b}-}-C_{\tau_{b}-}\right)^{+} \Lambda
$$

at $\tau_{b}$ if $\tau_{b}<\bar{\tau}^{\delta}$. Here the process $(-C)$ represents the amount of collateral funded by the bank, so that $\left(-\mathcal{W}_{\tau_{b^{-}}}-C_{\tau_{b^{-}}}\right)^{+}$represents the funding debt of the bank right before $\tau_{b} ; \Lambda \in[0,1]$ corresponds to the fractional loss of the funder in case of default of the bank, where we call funder a risk-free third party insuring funding of the trading strategy of the bank.

In Sect.5 we will provide a typical specification of all the data, in particular $\chi, g$ and $C$.

Let $(\Pi, \zeta)$ denote a to-be-determined price-and-hedge for the bank. After having bought the contract from the counterparty at time 0 at some price $\Pi_{0}$, the bank sets up a hedge $(-\zeta)$, which is a left-continuous row-vector process of the same dimension as $\mathcal{M}$.

We write $J=\mathbb{1}_{\llbracket 0, \tau \llbracket}, J^{\star}=\mathbb{1}_{\llbracket 0, \tau^{\star} \llbracket}$. 
Lemma 2.1 (Dynamics of the wealth process of the bank) Ignoring the close-out cashflow $\chi$ at $\bar{\tau}^{\delta}$ if $\tau<T$, which will be added separately later (see Lemma 3.2), we have $\mathcal{W}_{0}=-\Pi_{0}$ and, for $0<t \leq \bar{\tau}^{\delta}$,

$$
\begin{aligned}
d \mathcal{W}_{t}= & r_{t} \mathcal{W}_{t} d t+J_{t} d D_{t}-J_{t}^{\star} g_{t}\left(-\mathcal{W}_{t}\right) d t \\
& -\left(-\mathcal{W}_{\tau_{b}-}-C_{\tau_{b}-}\right)^{+} \Lambda \mathbb{1}_{\left\{\tau^{\star}=\tau_{b}<T\right\}} d J_{t}^{\star}-\zeta_{t} d \mathcal{M}_{t}
\end{aligned}
$$

Proof Collecting all terms in the above-described collateralization, hedging and funding scheme, we obtain $\mathcal{W}_{0}=-\Pi_{0}$ and, for $0<t \leq \bar{\tau}^{\delta}$ :

$$
\begin{aligned}
d \mathcal{W}_{t}= & \underbrace{J_{t} d D_{t}}_{\text {bank gets dividends }}-\underbrace{\zeta_{t} d \mathcal{M}_{t}}_{\text {hedging loss }} \\
& +\underbrace{J_{t}^{\star}\left(r_{t} \mathcal{W}_{t}-g_{t}\left(-\mathcal{W}_{t}\right)\right) d t}_{\text {funding benefits / costs to bank }} \\
& -\underbrace{(\underbrace{\star}}_{\left(-\mathcal{W}_{\tau_{b}-}-C_{\tau_{b}-}\right)^{+} \Lambda \mathbb{1}_{\left\{\tau^{\star}=\tau_{b}<T\right\}} d J_{t}^{\star}}
\end{aligned}
$$

windfall funding benefit of the bank at its own default time $\tau_{b}$ if $\tau_{b} \leq \tau_{c}^{\delta}$

$+$

$$
\underbrace{\left(1-J_{t}^{\star}\right) r_{t} \mathcal{W}_{t} d t}
$$

risk-free funding benefits/costs of the liquidator during the cure period

which yields (2.4).

\section{Full TVA BSDE}

In this section we derive the TVA BSDE with respect to the full model filtration $\mathbb{G}$.

\subsection{Risky price}

The risky price $\Pi$ is the value of the contract inclusive of counterparty and funding risk (as opposed to the risk-free price $P$ ). The justification for the following definition is provided by Lemma 3.2 below.

Definition 3.1 A risky price of the contract for the bank is a $(\mathbb{G}, \mathbb{Q})$ semimartingale $\Pi$ that satisfies the following price BSDE on $\llbracket 0, \bar{\tau}^{\delta} \rrbracket$ :

$$
\begin{aligned}
\Pi_{\bar{\tau}^{\delta}}= & \mathbb{1}_{\{\tau<T\}} \chi \\
d \nu_{t}:= & d \Pi_{t}-r_{t} \Pi_{t} d t \\
& +\left(\Pi_{\tau_{b}-}-C_{\tau_{b}-}\right)^{+} \Lambda \mathbb{1}_{\left\{\tau^{\star}=\tau_{b}<T\right\}} d J_{t}^{\star}+J_{t} d D_{t}-J_{t}^{\star} g_{t}\left(\Pi_{t}\right) d t
\end{aligned}
$$

defines a $(\mathbb{G}, \mathbb{Q})$ local martingale on $\llbracket 0, \bar{\tau}^{\delta} \rrbracket$. 
Lemma 3.2 If a risky price $\Pi$ can be found with $d v_{t}=\zeta_{t} d \mathcal{M}_{t}$ for some hedge $\zeta$, assuming $g_{t}(\pi)$ Lipschitz in $\pi$ with a Lipschitz constant that is Lebesgue integrable on $[0, T](\omega$-wise $)$, then $\left(\Pi_{0}, \zeta\right)$ yields a replication price and hedge for the bank, i.e., the resulting wealth process of the bank satisfies $\mathcal{W}=-\Pi$ on $\llbracket 0, \bar{\tau} \rrbracket \rrbracket$. In particular, we have

$$
\mathcal{W}_{\bar{\tau}^{\delta}}=-\Pi_{\bar{\tau}^{\delta}}=-\mathbb{1}_{\{\tau<T\}} \chi,
$$

so that after the close-out cashflow $\mathbb{1}_{\{\tau<T\}} \chi$ has been paid to the bank (or its liquidator if $\tau<T$ and $\tau_{b} \leq \tau_{c}^{\delta}$ ) at time $\bar{\tau}^{\delta}$, the bank's position is closed break-even.

Proof Under the assumptions of the lemma, the process $Z=\beta \Pi+\beta \mathcal{W}$ satisfies $Z_{0}=0$ and $d Z_{t}=\alpha_{t} Z_{t} d t$ on $\llbracket 0, \bar{\tau}^{\star} \llbracket$, where $\alpha_{t}:=\mathbb{1}_{\left\{\Pi_{t} \neq \mathcal{W}_{t}\right\}} \frac{g_{t}\left(\Pi_{t}\right)-g_{t}\left(-\mathcal{W}_{t}\right)}{\Pi_{t}-\left(-\mathcal{W}_{t}\right)}$ is Lebesgue integrable over $[0, T]$. Hence,

$$
d\left(e^{-\int_{0}^{t} \alpha_{s} d s} Z_{t}\right)=e^{-\int_{0}^{t} \alpha_{s} d s}\left(d Z_{t}-\alpha_{t} Z_{t} d t\right)=0,
$$

i.e., $e^{-\int_{0}^{t} \alpha_{s} d s} Z_{t}$ is constant on $\llbracket 0, \bar{\tau}^{\star} \llbracket$, equal to 0 in view of the initial condition for $Z$, i.e., $\mathcal{W}=-\Pi$ holds on $\llbracket 0, \bar{\tau}^{\star} \llbracket$. If $\tau<T$ and $\tau_{b} \leq \tau_{c}^{\delta}$, then this is followed by a jump of the two processes $\mathcal{W}$ and $(-\Pi)$ by the same amount $(2.3)$ at $\tau_{b}=\tau^{\star}=\bar{\tau}^{\star}$. In any case, $\mathcal{W}$ and $(-\Pi)$ coincide on $\llbracket 0, \bar{\tau}^{\star} \rrbracket$, and then again on $\llbracket \bar{\tau}^{\star}, \bar{\tau}^{\delta} \rrbracket$ by an argument similar to the one used in the beginning of the proof. Hence, $\mathcal{W}=-\Pi$ holds on $\llbracket 0, \bar{\tau} \delta \rrbracket$.

More broadly, if a risky price can be found with $d \nu_{t}=\zeta_{t} d \mathcal{M}_{t}+d \varepsilon_{t}$ for some hedge $\zeta$ and a "small" cost martingale $\varepsilon$, then the hedging error $\rho=\mathcal{W}+\Pi$, which starts from 0 at time 0 , remains "small" all the way through. In particular,

$$
\mathcal{W}_{\bar{\tau} \delta} \approx-\Pi_{\bar{\tau} \delta}=-\mathbb{1}_{\{\tau<T\}} \chi,
$$

so that after the close-out cashflow $\mathbb{1}_{\{\tau<T\}} \chi$ at $\bar{\tau}^{\delta}$, the bank's position is closed with a "small" hedging error.

3.2 Total valuation adjustment

Let

$$
Q_{t}=P_{t}+\Delta_{t}, \text { where } \beta_{t} \Delta_{t}=\int_{[\tau, t]} \beta_{s} d D_{s}
$$

(in particular, $\Delta_{t}=0$ and $Q_{t}=P_{t}$ for $t<\tau$ ). In words, $\Delta_{t}$ represents the cumulative contractual dividends capitalized at the risk-free rate that fail to be paid by the counterparty to the bank from time $\tau$ onwards. Hence $\Delta_{t}$ belongs to $Q_{t}$, the algebraic debt of the counterparty to the bank (before consideration of the collateral).

Definition 3.3 Given a risky price $\Pi$, the corresponding TVA is the process defined on $\llbracket 0, \bar{\tau}^{\delta} \rrbracket$ as $\Theta=Q-\Pi$. 
Let

$$
f_{t}(\vartheta)=g_{t}\left(Q_{t}-\vartheta\right)-r_{t} \vartheta \quad(\vartheta \in \mathbb{R})
$$

and

$$
\xi=Q_{\tau^{\delta}}-\chi, \quad \bar{\xi}_{t}=\mathbb{E}\left(\beta_{t}^{-1} \beta_{\tau^{\delta}} \xi \mid \mathcal{G}_{t}\right)\left(t \leq \bar{\tau}^{\delta}\right),
$$

assuming integrability of the so-called counterparty risk exposure $\xi$.

Lemma 3.4 Let there be given $\mathbb{G}$ semimartingales $\Pi$ and $\Theta$ such that $\Theta=Q-\Pi$ on $\llbracket 0, \bar{\tau}^{\delta} \rrbracket$. The process $\Pi$ is a risky price of the contract for the bank if and only if the process $\Theta$ satisfies the following $(\mathbb{G}, \mathbb{Q})$ TVA BSDE on $\llbracket 0, \bar{\tau}^{\delta} \rrbracket$ :

$$
\begin{aligned}
\Theta_{\bar{\tau}^{\delta}}= & \mathbb{1}_{\{\tau<T\}} \xi, \\
d \mu_{t}= & d \Theta_{t}-r_{t} \Theta_{t} d t+J_{t}^{\star} g_{t}\left(Q_{t}-\Theta_{t}\right) d t \\
& +\left(Q_{\tau_{b}-}-\Theta_{\tau_{b}-}-C_{\tau_{b^{-}}}\right)^{+} \Lambda \mathbb{1}_{\left\{\tau^{\star}=\tau_{b}<T\right\}} d J_{t}^{\star}
\end{aligned}
$$

defines $a(\mathbb{G}, \mathbb{Q})$ local martingale on $\llbracket 0, \bar{\tau}^{\delta} \rrbracket$.

Proof Assuming $\Theta$ defined in terms of a risky price $\Pi$ as $(Q-\Pi)$ on $\llbracket 0, \bar{\tau}^{\delta} \rrbracket$, the terminal condition for $\Theta$ in $(3.3)$ follows, by definition of the exposure $\xi$ in the left-hand side of 3.2 , from the terminal condition for $\Pi$ in 3.1]. Moreover, for $t \in \llbracket 0, \bar{\tau}^{\delta} \rrbracket$, we have

$$
-\beta_{t} \Theta_{t}=-\beta_{t} Q_{t}+\beta_{t} \Pi_{t}=-\left(\beta_{t} P_{t}+\int_{0}^{t} \beta_{s} d D_{s}\right)+\left(\beta_{t} \Pi_{t}+\int_{0}^{t} \beta_{s} J_{s} d D_{s}\right) .
$$

Hence,

$$
\begin{aligned}
& -\beta_{t} \Theta_{t}-\int_{0}^{t} \beta_{s} J_{s}^{\star} g_{s}\left(Q_{s}-\Theta_{s}\right) d s \\
& \quad+\int_{0}^{t} \beta_{\tau_{b}}\left(Q_{\tau_{b}-}-\Theta_{\tau_{b}-}-C_{\tau_{b}-}\right)^{+} \Lambda \mathbb{1}_{\left\{\tau^{\star}=\tau_{b}<T\right\}} d J_{s}^{\star} \\
& =-\left(\beta_{t} P_{t}+\int_{0}^{t} \beta_{s} d D_{s}\right)+\beta_{0} \Pi_{0}+\int_{0}^{t} d\left(\beta_{s} \Pi_{s}\right) \\
& \quad+\int_{0}^{t}\left(\beta_{\tau_{b}}\left(\Pi_{\tau_{b}-}-C_{\tau_{b}-}\right)^{+} \Lambda \mathbb{1}_{\left\{\tau^{\star}=\tau_{b}<T\right\}} d J_{s}^{\star}+\beta_{s} J_{s} d D_{s}-\beta_{s} J_{s}^{\star} g_{s}\left(\Pi_{s}\right) d s\right) \\
& =-\left(\beta_{t} P_{t}+\int_{0}^{t} \beta_{s} d D_{s}\right)+\beta_{0} \Pi_{0}+\int_{0}^{t} \beta_{s} d v_{s}
\end{aligned}
$$

(cf. (3.1)). Since $\left(\beta P+\int_{0}^{\cdot} \beta_{s} d D_{s}\right)$ (cf. $(2.1)$ ) and $v$ are $(\mathbb{G}, \mathbb{Q})$ local martingales, this establishes the martingale condition in 3.3 . Hence, 3.1) implies 3.3. The converse implication is proven similarly. 
As reflected in (3.3), the TVA problem, just like the risky pricing problem 3.1 is originally posed over the domain $\llbracket 0, \bar{\tau}^{\delta} \rrbracket$, which corresponds to the union of the three subdomains in Figure 3.1. with respective dividend and funding data abbreviated as $(D, g),(0, g)$ and $(0,0)$. But since data $(0,0)$ simply means, in terms of pricing, taking conditional expectation of the terminal condition discounted at the risk-free rate, the proposition that follows shows that the BSDE (3.3) can be reformulated as the BSDE (3.4) on the smaller time interval $\llbracket 0, \bar{\tau}^{\star} \rrbracket \subseteq \llbracket 0, \bar{\tau}^{\delta} \rrbracket$, modulo a modified terminal condition $\bar{\xi}$ at $\bar{\tau}^{\star}$ instead of $\xi$ at $\bar{\tau}^{\delta}$.

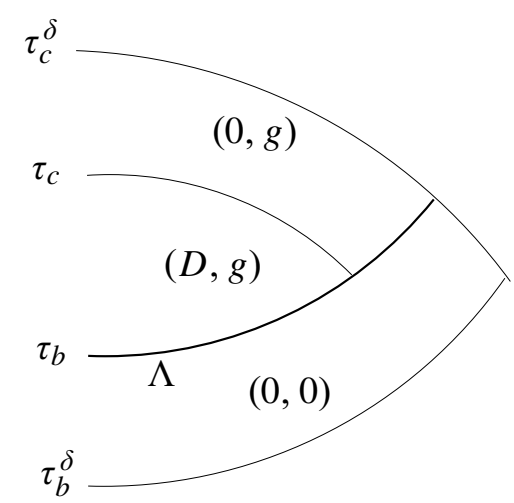

Fig. 3.1 Representation of the data of the TVA problem in a $(t, \omega)$ state space representation, focusing on the default and liquidation times and ignoring $T$ to alleviate the picture, i.e., "for $T=\infty$ ". The data in parentheses refer to the effective dividends and funding costs of the bank depending on the time $t$ and scenario $\omega$.

Proposition 3.5 Let there be given $\mathbb{G}$ semimartingales $\Pi$ and $\Theta$ such that $\Theta=Q-\Pi$ on $\llbracket 0, \bar{\tau}^{\delta} \rrbracket$. The process $\Pi$ is a risky price of the contract for the bank if and only if $\Theta=\mathbb{1}_{\{\tau<T\}} \bar{\xi}$ on $\rrbracket \bar{\tau}^{\star}, \bar{\tau}^{\delta} \rrbracket$ and $\Theta$ satisfies the following $(\mathbb{G}, \mathbb{Q})$ "exact TVA BSDE" on $\llbracket 0, \bar{\tau}^{\star} \rrbracket$ :

$$
\begin{aligned}
& \Theta_{\bar{\tau}^{\star}}=\mathbb{1}_{\{\tau<T\}}\left(\bar{\xi}_{\tau^{\star}}-\left(Q_{\tau_{b}-}-C_{\tau_{b}-}-\Theta_{\tau_{b}-}\right)^{+} \mathbb{1}_{\left\{\tau^{\star}=\tau_{b}\right\}} \Lambda\right) \text { and } \\
& d \mu_{t}:=d \Theta_{t}+f_{t}\left(\Theta_{t}\right) d t \text { is a }(\mathbb{G}, \mathbb{Q}) \text { local martingale on } \llbracket 0, \bar{\tau}^{\star} \rrbracket .
\end{aligned}
$$

Proof As explained before the proposition, (3.3) is equivalent to $\Theta=\mathbb{1}_{\{\tau<T\}} \bar{\xi}$ on $\llbracket \bar{\tau}, \bar{\tau}^{\delta} \rrbracket$ and (3.4) on $\llbracket 0, \bar{\tau} \rrbracket$.

Remark 3.6 This equivalence holds up to the value of $\Theta$ at $\tau^{\star}$ if $\tau^{\star}=\tau_{b}<T$, with a windfall funding benefit at default $\left(Q_{\tau_{b}-}-C_{\tau_{b}-}-\Theta_{\tau_{b}-}\right)^{+} \Lambda \mathbb{1}_{\left\{\tau=\tau_{b}<T\right\}}$ considered as a dividend at the intermediate time $\tau_{b}$ in (3.3) and as part of the valuation adjustment at the terminal time $\tau_{b}=\tau^{\star}$ in (3.4). Since the bank only risk manages $\Theta$ before $\tau_{b}$, this difference is immaterial in practice.

Because the cure period $\delta$ is only a few days, the quantitative impact on the TVA $\Theta$ of the coefficient $g$ on the time interval $\llbracket \bar{\tau}, \bar{\tau}^{\star} \rrbracket$ can only be very limited. Hence, for 
simplicity, we work henceforth with the following "full TVA BSDE":

$$
\begin{aligned}
\Theta_{\bar{\tau}} & =\mathbb{1}_{\{\tau<T\}}\left(\bar{\xi}_{\tau}-\left(P_{\tau_{b}-}-C_{\tau_{b}-}-\Theta_{\tau_{b}-}\right)^{+} \mathbb{1}_{\left\{\tau=\tau_{b}\right\}} \Lambda\right) \text { and } \\
d \mu_{t} & :=d \Theta_{t}+f_{t}\left(\Theta_{t}\right) d t \text { is a }(\mathbb{G}, \mathbb{Q}) \text { local martingale on } \llbracket 0, \bar{\tau} \rrbracket .
\end{aligned}
$$

This equation is obtained by replacing $g$ and $\Lambda$ by 0 on the time interval $\llbracket \bar{\tau}, \bar{\tau}^{\star} \rrbracket$ in the exact TVA BSDE (3.4), which means replacing $(0, g)$ by $(0,0)$ and $\Lambda$ by 0 in the upper right subdomain in Figure 3.1. Indeed, proceeding in this way, the argument already used for deriving Proposition 3.5 shows that the time interval on which the TVA needs be computed can be reduced further, from $\llbracket 0, \bar{\tau}^{\delta} \rrbracket$ initially to $\llbracket 0, \bar{\tau}^{\star} \rrbracket$ as done in Proposition 3.5 regarding the exact TVA BSDE, to even $\llbracket 0, \bar{\tau} \rrbracket$ now for the appropriately modified terminal condition regarding the full TVA BSDE (3.5), with all data set to zero outside $\llbracket 0, \bar{\tau} \rrbracket$. Reformulating the problem on the smaller domain $\llbracket 0, \bar{\tau} \rrbracket$ makes it simpler in view of the reduced-form analysis of Sect. 4 . because $\tau$, as opposed to $\tau^{\star}$, has an intensity.

\section{Reduced TVA BSDE}

Even if a bit simpler than (3.4), (3.5) is still a nonstandard BSDE. In this section we reduce the $(\mathbb{G}, \mathbb{Q})$ BSDE $(3.5)$ to the BSDE $(4.3)$ with a null terminal condition at the fixed time horizon $T$, stated with respect to a smaller filtration $\mathbb{F}$ and a possibly changed probability measure $\mathbb{P}$.

By Corollary 3.23 2) in He et al. [20], for any $\mathcal{G}_{\tau}$-measurable random variable $\kappa$, there exists a $\mathbb{G}$ predictable process $\widehat{\kappa}$ such that

$$
\mathbb{1}_{\{\tau<\infty\}} \mathbb{E}\left[\kappa \mid \mathcal{G}_{\tau-}\right]=\mathbb{1}_{\{\tau<\infty\}} \widehat{\kappa}_{\tau} .
$$

If $\kappa$ is integrable, then the time integral $\gamma_{t} \widehat{\kappa}_{t} d t$ exists (and is independent of the choice of a version of $\widehat{\kappa}$ ) and

$$
\kappa_{t} d J_{t}+\gamma_{t} \widehat{\kappa}_{t} d t
$$

is a $\left(\mathbb{G}, \mathbb{Q}\right.$ ) local martingale on $\mathbb{R}_{+}$(cf. Corollary 5.31 1) in He et al. [20]). In particular let $\widehat{\xi}$ be a $\mathbb{G}$ predictable process such that, on $\{\tau<\infty\}$,

$$
\widehat{\xi}_{\tau}=\mathbb{E}\left(\bar{\xi}_{\tau} \mid \mathcal{G}_{\tau-}\right)=\mathbb{E}\left(\beta_{\tau}^{-1} \beta_{\tau+\delta} \xi \mid \mathcal{G}_{\tau-}\right) .
$$

For $t \in \llbracket 0, \bar{\tau} \rrbracket$ and $\vartheta \in \mathbb{R}$, we write

$$
\begin{aligned}
\widehat{f_{t}}(\vartheta) & =f_{t}(\vartheta)+\gamma_{t} \widehat{\xi}_{t}-\left(P_{t}-C_{t}-\vartheta\right)^{+} \gamma_{t}^{b} \Lambda-\gamma_{t} \vartheta \\
& =\gamma_{t} \widehat{\xi}_{t}+g_{t}\left(P_{t}-\vartheta\right)-\left(P_{t}-C_{t}-\vartheta\right)^{+} \gamma_{t}^{b} \Lambda-\left(r_{t}+\gamma_{t}\right) \vartheta .
\end{aligned}
$$

For any left-limited process $Y$, we denote by $Y^{\tau-}=J Y+(1-J) Y_{-}$the process $Y$ stopped at $(\tau-)$. Let $S$ denote the Azéma supermartingale of $\tau$, i.e., the process such that $S_{t}=\mathbb{Q}\left(\tau>t \mid \mathcal{F}_{t}\right), t \geq 0$. Conditions of the following kind are studied at the theoretical level in Crépey and Song (2015a, 2015b).

Condition (B). $\mathbb{F}$ is a subfiltration of $\mathbb{G}$ satisfying the usual conditions such that any $\mathbb{G}$ predictable process $U$ admits an $\mathbb{F}$ predictable reduction, i.e., an $\mathbb{F}$ predictable process, denoted by $U^{\prime}$, that coincides with $U$ on $\rrbracket 0, \tau \rrbracket$. 
Remark 4.1 If $S_{T}>0$ holds almost surely, then any inequality between two $\mathbb{G}$ predictable processes on $\rrbracket 0, \tau \rrbracket$ implies the same inequality between their $\mathbb{F}$ predictable reductions on $(0, T]$ (see Song [25, Lemma 6.1]); In particular (see also Crépey and Song [13, Lemma A.1]), the process $U^{\prime}$ is uniquely determined on $(0, T]$.

Condition (C). There exist:

(C.1) A subfiltration $\mathbb{F}$ of $\mathbb{G}$ satisfying the usual conditions such that $\mathbb{F}$ semimartingales stopped at $\tau$ are $\mathbb{G}$ semimartingales;

(C.2) A probability measure $\mathbb{P}$ equivalent to $\mathbb{Q}$ on $\mathcal{F}_{T}$ such that $(\mathbb{F}, \mathbb{P})$ local martingales stopped at $(\tau-)$ are $(\mathbb{G}, \mathbb{Q})$ local martingales on $[0, T]$;

(C.3) An $\mathbb{F}$ progressive reduction $\widetilde{f}_{t}(\vartheta)$ of $\widehat{f_{t}}(\vartheta)$, i.e., an $\mathcal{R}(\mathbb{F}) \otimes \mathcal{B}(\mathbb{R})$ function $\widetilde{f}_{t}(\vartheta)$ such that $\int_{0}^{\cdot} \widehat{f_{t}}(\vartheta) d t=\int_{0}^{\cdot} \widetilde{f}_{t}(\vartheta) d t$ on $\llbracket 0, \bar{\tau} \rrbracket$.

The condition $(\mathrm{C})$ is much more versatile than a standard immersion reduced-form intensity model of credit risk, where $\mathbb{P}$ has to be taken equal to $\mathbb{Q}$ and where the full model filtration $\mathbb{G}$ has to be taken as the reference filtration $\mathbb{F}$ progressively enlarged by $\tau$ (see Bielecki et al. [4]). In particular, even for $\mathbb{P}=\mathbb{Q}$, the conditions (C.1-2-3) do not exclude a jump of an $\mathbb{F}$ adapted càdlàg process at time $\tau$. This happens for instance with a nonvanishing "gap dividend" $\Delta_{\tau}=D_{\tau}-D_{\tau-}$ at time $\tau$ in the DMO model of Sect.77. By contrast, a jump of an $\mathbb{F}$ adapted càdlàg process at time $\tau$ cannot happen in a basic reduced-form credit risk setup (see Crépey et al. [10, Lemma 13.7.3(ii)]).

The result that follows can also be retrieved from Crépey and Song [12], but in a much more abstract setup there, mainly motivated by the converse to this result. Hence, we provide a self-contained proof, valid under the "minimal condition" (C).

Theorem 4.2 Under the condition $(C)$, assume that an $(\mathbb{F}, \mathbb{P})$ semimartingale $\widetilde{\Theta}$ satisfies the following "reduced TVA BSDE" on $[0, T]$ :

$$
\begin{aligned}
\widetilde{\Theta}_{T} & =0 \text { and } \\
d \widetilde{\mu}_{t} & :=d \widetilde{\Theta}_{t}+\widetilde{f}_{t}\left(\widetilde{\Theta}_{t}\right) d t \text { is an }(\mathbb{F}, \mathbb{P}) \text { local martingale on }[0, T] .
\end{aligned}
$$

Let $\Theta=\widetilde{\Theta}$ on $\llbracket 0, \bar{\tau} \llbracket$ and $\Theta_{\bar{\tau}}=\mathbb{1}_{\{\tau<T\}}\left(\bar{\xi}_{\tau}-\left(P_{\tau-}-C_{\tau-}-\widetilde{\Theta}_{\tau-}\right)^{+} \mathbb{1}_{\left\{\tau=\tau_{b}\right\}} \Lambda\right)$. Then $\Theta$ is a $(\mathbb{G}, \mathbb{Q})$ semimartingale satisfying the full TVA BSDE 3.5 on $\llbracket 0, \bar{\tau} \rrbracket$ and we have, for $t \in \llbracket 0, \bar{\tau} \rrbracket$,

$$
\begin{aligned}
d \mu_{t}= & d \widetilde{\mu}_{t}^{\tau-}-\left(\bar{\xi}_{\tau}-\left(P_{\tau-}-C_{\tau-}-\widetilde{\Theta}_{\tau-}\right)^{+} \mathbb{1}_{\left\{\tau=\tau_{b}\right\}} \Lambda-\widetilde{\Theta}_{\tau-}\right) d J_{t} \\
& -\left(\left(\widehat{\xi}_{t}-\widetilde{\Theta}_{t}\right) \gamma_{t}-\left(P_{t}-C_{t}-\widetilde{\Theta}_{t}\right)^{+} \gamma_{t}^{b} \Lambda\right) d t
\end{aligned}
$$

Proof By definition of $\Theta$ here, a $(\mathbb{G}, \mathbb{Q})$ semimartingale by (C.1), we have, for $t \in \llbracket 0, \bar{\tau} \rrbracket:$

$$
\begin{aligned}
d \Theta_{t} & =d\left(J_{t} \widetilde{\Theta}_{t}\right)-\left(\bar{\xi}_{\tau}-\left(P_{\tau-}-C_{\tau-}-\widetilde{\Theta}_{\tau-}\right)^{+} \mathbb{1}_{\left\{\tau=\tau_{b}\right\}} \Lambda\right) d J_{t} \\
& =d \widetilde{\Theta}_{t}^{\tau-}+\widetilde{\Theta}_{\tau-} d J_{t}-\left(\bar{\xi}_{\tau}-\left(P_{\tau-}-C_{\tau-}-\widetilde{\Theta}_{\tau-}\right)^{+} \mathbb{1}_{\left\{\tau=\tau_{b}\right\}} \Lambda\right) d J_{t} .
\end{aligned}
$$


Then by 4.3 , for $t \in \llbracket 0, \bar{\tau} \rrbracket$ :

$$
\begin{aligned}
-d \Theta_{t}= & \widetilde{f}_{t}\left(\widetilde{\Theta}_{t}\right) d t-d \widetilde{\mu}_{t}^{\tau-}+\left(\bar{\xi}_{\tau}-\left(P_{\tau-}-C_{\tau-}-\widetilde{\Theta}_{\tau-}\right)^{+} \mathbb{1}_{\left\{\tau=\tau_{b}\right\}} \Lambda-\widetilde{\Theta}_{\tau-}\right) d J_{t} \\
= & f_{t}\left(\Theta_{t}\right) d t-d \widetilde{\mu}_{t}^{\tau-}+\left(\bar{\xi}_{\tau}-\left(P_{\tau-}-C_{\tau-}-\widetilde{\Theta}_{\tau-}\right)^{+} \mathbb{1}_{\left\{\tau=\tau_{b}\right\}} \Lambda-\widetilde{\Theta}_{\tau-}\right) d J_{t} \\
& \left.+\left(\widetilde{\xi}_{t}-\widetilde{\Theta}_{t}\right) \gamma_{t}-\left(P_{t}-C_{t}-\Theta_{t}\right)^{+} \gamma_{t}^{b} \Lambda\right) d t,
\end{aligned}
$$

by $(\mathrm{C} .3)$. By $(\mathrm{C} .2), \tilde{\mu}_{t}^{\tau-}$ is a $(\mathbb{G}, \mathbb{Q})$ local martingale, as is also on $\llbracket 0, \bar{\tau} \rrbracket$

$$
\begin{aligned}
\left(\bar{\xi}_{\tau}-\left(P_{\tau-}-C_{\tau-}-\widetilde{\Theta}_{\tau-}\right)^{+} \mathbb{1}_{\left\{\tau=\tau_{b}\right\}} \Lambda-\widetilde{\Theta}_{\tau-}\right) d J_{t} \\
\left.+\left(\widehat{\xi}_{t}-\widetilde{\Theta}_{t}\right) \gamma_{t}-\left(P_{t}-C_{t}-\Theta_{t}\right)^{+} \gamma_{t}^{b} \Lambda\right) d t
\end{aligned}
$$

(cf. (4.1)). This yields the decomposition (4.4) of $d \mu_{t}:=d \Theta_{t}+f_{t}\left(\Theta_{t}\right) d t$, which implies the martingale condition in [3.5], where the terminal condition holds by definition of $\Theta_{\bar{\tau}}$ in the theorem.

\section{Well-posedness of the TVA BSDEs}

In applications, we need to specify the close-out cashflow $\chi$, hence the counterparty risk exposure $\xi=Q_{\tau^{\delta}}-\chi$, and the funding coefficient $g_{t}(\pi)$. Let $V$ denote the variation margin process, where $V \geq 0$ (resp. $\leq 0$ ) means collateral posted by the counterparty and received by the bank (resp. posted by the bank and received by the counterparty). Let processes $I^{c} \geq 0$ and $I^{b} \leq 0$ represent the initial margin posted by the counterparty and the negative of the initial margin posted by the bank. Let

$$
C^{c}=V+I^{c} \text { and } C^{b}=V+I^{b},
$$

which correspond to the total collateral guarantee for the bank and the negative of the total collateral guarantee for the counterparty. We assume that all the margin processes are stopped at $\tau$. The bank's close-out cashflow $\chi$ is derived from the liquidation debts of the counterparty to the bank and vice versa, respectively modelled at time $\tau^{\delta}$ as

$$
\varepsilon_{c}=\left(Q_{\tau^{\delta}}-C_{\tau}^{c}\right)^{+}, \varepsilon_{b}=\left(Q_{\tau^{\delta}}-C_{\tau}^{b}\right)^{-} .
$$

Note that $\varepsilon_{c} \times \varepsilon_{b} \equiv 0$, by nonnegativity of $I^{c}$ and $\left(-I^{b}\right)$. The close-out cashflow is modelled as

$$
\chi=\left\{\begin{array}{c}
C_{\tau}^{c}+R_{c} \varepsilon_{c} \text { if } \varepsilon_{c}>0 \text { and } \tau_{c} \leq \tau_{b}^{\delta}, \\
C_{\tau}^{b}-R_{b} \varepsilon_{b} \text { if } \varepsilon_{b}>0 \text { and } \tau_{b} \leq \tau_{c}^{\delta}, \\
Q_{\tau^{\delta}} \text { otherwise. }
\end{array}\right.
$$

Here $R_{c}$ and $R_{b}$ stand for constant recovery rates of the counterparty to the bank and vice versa. The ensuing counterparty risk exposure of the bank results from the left-hand side in 3.2) as

$$
\xi=Q_{\tau^{\delta}}-\chi=\mathbb{1}_{\left\{\tau_{c} \leq \tau_{b}^{\delta}\right\}}\left(1-R_{c}\right) \varepsilon_{c}-\mathbb{1}_{\left\{\tau_{b} \leq \tau_{c}^{\delta}\right\}}\left(1-R_{b}\right) \varepsilon_{b} .
$$

We assume that the posted collateral is remunerated at a rate $\left(r_{t}+c_{t}\right)$ and that the bank can invest cash at a rate $\left(r_{t}+\lambda_{t}\right)$ and obtain unsecured funding at a rate 
$\left(r_{t}+\bar{\lambda}_{t}\right)$, for $\mathbb{G}$ predictable processes $c, \lambda, \bar{\lambda}$ (and also $r$, noting that predictability is not a true restriction with respect to progressive measurability for all these processes that are time integrated). Assuming all the margins re-hypothecable, i.e., reusable by the receiving party, then the collateral funded by the bank is $(-C)$, where

$$
C=C^{b}+I^{c}=V+I^{c}+I^{b} .
$$

This results in the following $d t$-funding cost of the bank, for $t<\bar{\tau}^{\star}$ :

$$
\underbrace{J_{t}\left(r_{t}+c_{t}\right) C_{t}}_{\text {remuneration of the collateral }}+\underbrace{\left(r_{t}+\bar{\lambda}_{t}\right)\left(-\mathcal{W}_{t}-C_{t}\right)^{+}-\left(r_{t}+\lambda_{t}\right)\left(-\mathcal{W}_{t}-C_{t}\right)^{-}}_{\text {funding costs / benefits }},
$$

which is of the form 2.2 with in particular, for $t<\tau$,

$$
g_{t}(\pi)=c_{t} C_{t}+\bar{\lambda}_{t}\left(\pi-C_{t}\right)^{+}-\lambda_{t}\left(\pi-C_{t}\right)^{-} .
$$

If part of the collateral is segregated (as opposed to re-hypothecable), then the detail changes slightly, but the overall structure (2.2) of the funding costs is still valid. Assuming henceforth (5.2), $\widehat{f_{t}}(\vartheta)$ in $(4.2)$ is such that, on $\llbracket 0, \bar{\tau} \rrbracket$,

$$
\begin{aligned}
\widehat{f_{t}}(\vartheta)+\left(r_{t}+\gamma_{t}\right) \vartheta= & \gamma_{t} \widehat{\xi}_{t}+c_{t} C_{t}+\bar{\lambda}_{t}\left(P_{t}-C_{t}-\vartheta\right)^{+} \\
& -\lambda_{t}\left(P_{t}-C_{t}-\vartheta\right)^{-}-\left(P_{t}-C_{t}-\vartheta\right)^{+} \gamma_{t}^{b} \Lambda \\
= & \underbrace{\gamma_{t} \widehat{\xi}_{t}}_{c d v a_{t}}+\underbrace{c_{t} C_{t}+\widetilde{\lambda}_{t}\left(P_{t}-C_{t}-\vartheta\right)^{+}-\lambda_{t}\left(P_{t}-C_{t}-\vartheta\right)^{-}}_{f v a_{t}(\vartheta)},
\end{aligned}
$$

where $\tilde{\lambda}_{t}=\bar{\lambda}_{t}-\gamma_{t}^{b} \Lambda$ can be interpreted as a liquidity borrowing spread of the bank, net of its credit spread to its funder. From the perspective of the bank, the term $\gamma_{t} \widehat{\xi}_{t}$ represents the counterparty risk component of $\widehat{f_{t}}(\vartheta)$, whereas the remaining terms are the risky funding components. The positive (resp. negative) components of $\widehat{f_{t}}(\vartheta)$ can be considered as deal adverse (resp. deal friendly) as they increase (resp. decrease) the TVA $\Theta$ of the bank. Depending on the sign of $\Pi=Q-\Theta$, a "less positive" $\Pi$ is interpreted as a lower buyer price by the bank and a "more negative" $\Pi$ as a higher seller price by the bank.

Note that the DVA cashflow $\mathbb{1}_{\left\{\tau_{b} \leq \tau_{c}^{\delta}\right\}}\left(1-R_{b}\right) \varepsilon_{b}$ in (5.1) and the "DVA2" cashflow (2.3) are benefits of the bank at its own default. The materiality of such windfall benefits has been the topic of intense debates among the quant and academic finance communities (see e.g. Hull and White [21]).

On $\llbracket 0, \bar{\tau} \rrbracket$, we have

$$
\begin{aligned}
\widehat{f_{t}}(\vartheta)= & \gamma_{t} \widehat{\xi}_{t}+c_{t} C_{t}+\tilde{\lambda}_{t}\left(P_{t}-C_{t}-\vartheta\right)^{+}-\lambda_{t}\left(P_{t}-C_{t}-\vartheta\right)^{-}-\left(r_{t}+\gamma_{t}\right) \vartheta \\
= & \gamma_{t} \widehat{\xi}_{t}+c_{t} C_{t}+\bar{\lambda}_{t}\left(P_{t}-C_{t}-\vartheta\right)^{+}-\lambda_{t}\left(P_{t}-C_{t}-\vartheta\right)^{-} \\
& -\gamma_{t}\left(P_{t}-C_{t}\right)+\left(\gamma_{t}-\gamma_{t}^{b} \Lambda\right)\left(P_{t}-C_{t}-\vartheta\right)^{+}-\gamma_{t}\left(P_{t}-C_{t}-\vartheta\right)^{-}-r_{t} \vartheta .
\end{aligned}
$$

Assuming the condition (B) and $S_{T}>0$ almost surely, we may and do henceforth choose for $\widetilde{f}_{t}(\vartheta)$ the process obtained from $\widehat{f_{t}}(\vartheta)$ by replacing each process $U$ involved in (5.4) by its $\mathbb{F}$ predictable reduction $U^{\prime}$ or, in the case of $U=P$ or $C$, by $\left(P_{-}\right)^{\prime}$ and $\left(C_{-}\right)^{\prime}$. We write $\|U\|_{\widetilde{\mathcal{H}}_{p}}^{p}=\widetilde{\mathbb{E}}\left[\int_{0}^{T} U_{t}^{p} d t\right](p>0)$, where $\widetilde{\mathbb{E}}$ means $\mathbb{P}$ expectation. 
The boundedness assumption on $P$ and $C$ in the following result is far from minimal, but it holds in the case of vanilla credit derivatives such as CDS contracts and CDO tranches considered later in the paper.

Theorem 5.1 Under the condition (B), assuming $S_{T}>0$ almost surely, $\lambda^{\prime}, \bar{\lambda}^{\prime}$ and $r^{\prime}$ bounded from below on $[0, T],\left(P_{-}\right)^{\prime}$ and $\left(C_{-}\right)^{\prime}$ bounded on $[0, T]$ and $c^{\prime}, \lambda^{\prime}, \bar{\lambda}^{\prime}, r^{\prime}$, $\gamma^{\prime}$ in $\widetilde{\mathcal{H}}_{2}$, then the reduced BSDE (4.3) (with $\widetilde{f}$ as specified above) is well posed in $\widetilde{\mathcal{H}}_{2}$, where well-posedness includes existence, uniqueness, comparison and the standard a priori bound and error BSDE estimates. The $\widetilde{\mathcal{H}}_{2}$ solution $\widetilde{\Theta}$ to 4.3 satisfies

$$
\widetilde{\Theta}_{t}=\widetilde{\mathbb{E}}\left[\int_{t}^{T} \widetilde{f}_{s}\left(\widetilde{\Theta}_{s}\right) d s \mid \mathcal{F}_{t}\right], t \in[0, T] .
$$

Proof Assuming $\lambda^{\prime}, \bar{\lambda}^{\prime}$ and $r^{\prime}$ bounded from below, it follows from (5.4) (where $\left.\gamma_{t} \geq \gamma_{t}^{b} \Lambda \geq 0\right)$ and from the remark 4.1 that $\widetilde{f}_{t}(\vartheta)$ satisfies the so-called monotonicity condition

$$
\left(\widetilde{f}_{t}(\vartheta)-\widetilde{f}_{t}\left(\vartheta^{\prime}\right)\right)\left(\vartheta-\vartheta^{\prime}\right) \leq C\left(\vartheta-\vartheta^{\prime}\right)^{2}
$$

on $(0, T]$, for some constant $C$. Moreover, we have

$$
|\tilde{f}(\vartheta)-\tilde{f}(0)| \leq\left(\left|\bar{\lambda}^{\prime}\right|+\left|\lambda^{\prime}\right|+\left|r^{\prime}\right|+\gamma^{\prime}\right)|\vartheta| .
$$

Hence, having assumed $\left(P_{-}\right)^{\prime},\left(C_{-}\right)^{\prime}$ bounded and $c^{\prime}, \lambda^{\prime}, \bar{\lambda}^{\prime}, r^{\prime}, \gamma^{\prime}$ in $\widetilde{\mathcal{H}}_{2}$, the following integrability conditions hold:

$$
\sup _{|\vartheta| \leq \bar{\vartheta}}|\widetilde{f} .(\vartheta)-\widetilde{f} .(0)| \in \widetilde{\mathcal{H}}_{1}(\bar{\vartheta}>0), \quad \widetilde{f} .(0) \in \widetilde{\mathcal{H}}_{2} .
$$

Therefore, by application of the results of Kruse and Popier [24, Sect. 4], the reduced BSDE (4.3) with coefficient $\widetilde{f}_{t}(\vartheta)$ is well posed in $\widetilde{\mathcal{H}}_{2}$, where well-posedness includes existence, uniqueness, comparison and the standard BSDE bound and error estimates. The identity $(5.5)$ is the usual integral representation for an $\widetilde{\mathcal{H}}_{2}$ solution $\widetilde{\Theta}$ to the reduced BSDE (4.3).

Under the conditions (C), (B) (which can be viewed as a reinforcement of (C.1) and (C.3)) and $S_{T}>0$ almost surely, the results of Crépey and Song [12] show that the reduced BSDE does not only imply the full BSDE (cf. Theorem 4.2 above), but is in fact equivalent to it. Theorem 5.1 establishes the well-posedness of the reduced BSDE (4.3) (under a typical specification of the data). Hence, the full BSDE 3.5 is well posed too.

\section{Marked default times setup}

From a practical point of view, Theorems 4.2 and 5.1 allow modelling a TVA process as a solution to the "simple", reduced TVA BSDE (4.3). A residual issue is the specification of a concrete but general enough framework where $c d v a=\gamma \widehat{\xi}$ in 5.3 can be computed in practice. Toward this aim, this section implements the reduced-form 
approach of the previous sections, based on a default time $\tau$ obtained as a $\mathbb{G}$ stopping time with a mark. The mark allows conveying some additional information about the default, such as wrong-way and gap risk features that would be out-of-reach in the basic immersion setup of Crépey [9].

We assume in the sequel that $\tau$ is endowed with a mark $e$ in a finite set $E$, i.e.,

$$
\tau=\min _{e \in E} \tau_{e}
$$

where the $\tau_{e}$ are $\mathbb{G}$ stopping times avoiding each other. We suppose that each time $\tau_{e}$ has a $(\mathbb{G}, \mathbb{Q})$ intensity $\gamma_{t}^{e}$ and that

$$
\mathcal{G}_{\tau}=\mathcal{G}_{\tau-} \vee \sigma(l)
$$

in which $l=\operatorname{argmin}_{e \in E} \tau_{e}$ is the identity of the mark of $\tau$.

Remark 6.1 The assumption of a finite set $E$ in 6.1 ensures tractability of the setup, while offering a sufficiently large playground for applications, as the second part of the paper demonstrates. This finiteness assumption could be removed at the cost of considering an integral instead of a sum over $E$ in 6.3 and similar expressions later in the paper.

We denote by $\mathcal{E}$ the powerset of $E$ and by $\lambda$ the Lebesgue measure on $\mathbb{R}_{+}$.

Lemma 6.2 Assuming a marked stopping time $\tau$ as above, then for any $\mathcal{G}_{\tau}$-measurable random variable $\kappa$, there exists a $\mathcal{P}(\mathbb{G}) \otimes \mathcal{E}$-measurable function $\widetilde{\kappa}=\widetilde{\kappa}_{t}^{e}$ such that

$$
\mathbb{1}_{\left\{\tau=\tau_{e}\right\}} \kappa=\mathbb{1}_{\left\{\tau=\tau_{e}\right\}} \widetilde{\kappa}_{\tau}^{e}, e \in E .
$$

For any such function $\widetilde{\kappa}, a \mathbb{Q} \times \lambda$ a.e. version of $\gamma \widehat{\kappa}$ is given by $J_{-} \sum_{E} \gamma^{e} \widetilde{\kappa}^{e}$. In particular, the intensity of $\tau$ satisfies $\gamma=J_{-} \sum_{e \in E} \gamma^{e}, \mathbb{Q} \times \lambda$ a.e., and we have

$$
c d v a=J_{-} \sum_{e \in E} \gamma^{e} \widetilde{\xi}^{e}, \mathbb{Q} \times \lambda \text { a.e. }
$$

for any $\mathcal{P}(\mathbb{G}) \otimes \mathcal{E}$-measurable function $\widetilde{\xi}=\widetilde{\xi}_{t}^{e}$, which exists, such that, for each $e \in E$,

$$
\bar{\xi}_{\tau}=\widetilde{\xi}_{\tau}^{e} \text { on the event }\left\{\tau=\tau_{e}\right\}
$$

Proof The existence of the processes $\widetilde{\kappa}^{e}(\pi)$ follows from $(6.2)$. By $\mathbb{G}$ predictability of these processes, the $\widetilde{\kappa}_{\tau}^{e}$ are $\mathcal{G}_{\tau-}$ locally integrable. Then, on $\{\tau<\infty\}$,

$$
\mathbb{E}\left[\kappa \mid \mathcal{G}_{\tau-}\right]=\mathbb{E}\left[\sum_{e \in E} \mathbb{1}_{\left\{\tau=\tau^{e}\right\}} \widetilde{\kappa}_{\tau}^{e} \mid \mathcal{G}_{\tau-}\right]=\sum_{e \in E} \widetilde{\kappa}_{\tau}^{e} \mathbb{E}\left[\mathbb{1}_{\left\{\tau=\tau^{e}\right\}} \mid \mathcal{G}_{\tau-}\right] .
$$

Let $q_{t}^{e}$ denote a $\mathcal{P}(\mathbb{G}) \otimes \mathcal{E}$-measurable function, which exists by Corollary 3.23 2) in He et al. [20], such that $q_{\tau}^{e} \mathbb{1}_{\{\tau<\infty\}}=\mathbb{E}\left[\mathbb{1}_{\left\{\tau=\tau^{e}\right\}} \mid \mathcal{G}_{\tau-}\right] \mathbb{1}_{\{\tau<\infty\}}(e \in E)$. For bounded $Z \in \mathcal{P}(\mathbb{G})$, we compute $\mathbb{E}\left[Z_{\tau} \mathbb{1}_{\left\{\tau=\tau^{e}<\infty\right\}}\right]$ in two ways:

$$
\mathbb{E}\left[Z_{\tau} \mathbb{1}_{\left\{\tau=\tau^{e}<\infty\right\}}\right]=\mathbb{E}\left[Z_{\tau} q_{\tau}^{e} \mathbb{1}_{\{\tau<\infty\}}\right]=\mathbb{E}\left[\int_{0}^{\infty} Z_{s} q_{s}^{e} \gamma_{s} d s\right],
$$


and

$$
\begin{aligned}
\mathbb{E}\left[Z_{\tau} \mathbb{1}_{\left\{\tau=\tau^{e}<\infty\right\}}\right] & =\mathbb{E}\left[Z_{\tau^{e}} \mathbb{1}_{\left\{\tau=\tau^{e}<\infty\right\}}\right] \\
& =\mathbb{E}\left[Z_{\tau^{e}} \mathbb{1}_{\left\{\tau^{e} \leq \tau<\infty\right\}}\right]=\mathbb{E}\left[\int_{0}^{\infty} Z_{s} \mathbb{1}_{\{s \leq \tau\}} \gamma_{s}^{e} d s\right] .
\end{aligned}
$$

Hence, $\mathbb{Q}$ almost surely, $q_{t}^{e} \gamma_{t}=\mathbb{1}_{\{t \leq \tau\}} \gamma_{t}^{e}$ holds $\lambda$ almost everywhere, so that

$$
\mathbb{Q}\left[q_{\tau}^{e} \gamma_{\tau} \neq \gamma_{\tau}^{e}, \tau<\infty\right]=\mathbb{E}\left[\mathbb{1}_{\left\{q_{\tau}^{e} \gamma_{\tau} \neq \gamma_{\tau}^{e}, \tau<\infty\right\}}\right]=\mathbb{E}\left[\int_{0}^{\infty} \mathbb{1}_{\left\{q_{t}^{e} \gamma_{t} \neq \gamma_{t}^{e}\right\}} \gamma_{t} d t\right]=0
$$

Therefore, on $\{\tau<\infty\}$,

$$
\gamma_{\tau} \widehat{\kappa}_{\tau}=\gamma_{\tau} \mathbb{E}\left[\kappa \mid \mathcal{G}_{\tau-}\right]=\sum_{e \in E} \widetilde{\kappa}_{\tau}^{e} \gamma_{\tau} q_{\tau}^{e}=\sum_{e \in E} \widetilde{\kappa}_{\tau}^{e} \gamma_{\tau}^{e}
$$

This implies that

$$
\gamma \widehat{\kappa}=J_{-} \sum_{E} \gamma^{e} \widetilde{\kappa}^{e}, \mathbb{Q} \times \lambda \text {-a.e. }
$$

In particular, 6.3 follows by definition 5.3 of $c d v a$.

We now provide a concrete specification ensuring (6.2), in the case where $\mathbb{G}$ is the progressive enlargement of a reference filtration $\mathbb{F}$ by $n$ random times $\eta_{1}, \ldots, \eta_{n}$ that avoid each other. Let the $\eta_{(i)}$ be the increasing ordering of the $\eta_{i}$, with also $\eta_{(0)}=0$ and $\eta_{(n+1)}=\infty$. The optional splitting formula of Song [26] states that, for any $\mathbb{G}$ optional process $Y$, there exist $\mathcal{O}(\mathbb{F}) \otimes \mathcal{B}\left([0, \infty]^{n}\right)$-measurable functions $Y^{(0)}, Y^{(1)}, \ldots, Y^{(n)}$ such that

$$
Y=\sum_{i=0}^{n} Y^{(i)}\left(\eta_{1} \nmid \eta_{(i)}, \ldots, \eta_{n} \nmid \eta_{(i)}\right) \mathbb{1}_{\left[\eta_{(i)}, \eta_{(i+1)}\right)},
$$

where $a \nmid b$ denotes $a$ if $a \leq b$ and $\infty$ if $a>b$, for $a, b \in[0, \infty]$. This formula holds, in particular, in any recursively immersed or multivariate density model of default times. By a recursively immersed model of default times, we mean a model where a reference filtration is successively progressively enlarged by random times, such that each successive enlargement has the immersion property. By a multivariate density model, we mean a model with a conditional density of the default times given some reference market filtration.

Lemma 6.3 Assuming the optional splitting formula in force, e.g. in any recursively immersed or multivariate density model of default times, let $\eta=\eta_{1} \wedge \eta_{2}$. If the $\eta_{i}$ avoid each other and that $\eta$ avoids $\mathbb{F}$ stopping times, then

$$
\mathcal{G}_{\eta}=\mathcal{G}_{\eta-} \vee \sigma\left(\left\{\eta=\eta_{1}\right\},\left\{\eta=\eta_{2}\right\}\right)
$$


Proof Let $N=\{1,2,3, \ldots, n\}$. By the optional splitting formula 6.5 , for any $\mathbb{G}$ optional process $Y$ and $i \in N$, we have

$$
\begin{aligned}
Y_{\eta} \mathbb{1}_{\left\{\eta=\eta_{(i)}\right\}} & =Y_{\eta}^{(i)}\left(\eta_{1} \nmid \eta, \ldots, \eta_{n} \nmid \eta\right) \mathbb{1}_{\left\{\eta=\eta_{(i)}\right\}} \\
& =\sum_{I \subseteq N ;|I|=i-1} Y_{\eta}^{(i)}\left(\eta_{1} \nmid \eta, \eta_{2} \nmid \eta, \ldots, \eta_{n} \nmid \eta\right) \mathbb{1}_{\left\{\forall j \in I, \eta_{j}<\eta\right\}} \mathbb{1}_{\left\{\forall j \in N \backslash I, \eta \leq \eta_{j}\right\}} \\
& =\sum_{k=1}^{2} \mathbb{1}_{\left\{\eta=\eta_{k}\right\}} \sum_{I \subseteq N ;|I|=i-1} Y_{\eta}^{(i, I, k)}\left(\eta ; \eta_{j}, j \in I\right) \mathbb{1}_{\left\{\forall j \in I, \eta_{j}<\eta\right\}} \mathbb{1}_{\left\{\forall j \in N \backslash I, \eta \leq \eta_{j}\right\}},
\end{aligned}
$$

where $Y_{t}^{(i, I, k)}\left(\omega, y ; y_{j}, j \in I\right)$ is $\mathcal{O}(\mathbb{F}) \otimes \mathcal{B}([0, \infty]) \otimes \mathcal{B}\left([0, \infty]^{i-1}\right)$-measurable. Moreover, as $\eta$ avoids $\mathbb{F}$ stopping times, He et al. [20, Theorem 3.20] and the monotone class theorem imply that $Y_{\eta}^{(i, I, k)}\left(\eta ; \eta_{j}, j \in I\right)$ is $\mathcal{G}_{\eta-}$-measurable. So, on each event $\left\{\eta=\eta_{k}\right\}, Y_{\eta} \mathbb{1}_{\left\{\eta=\eta_{(i)}\right\}}$ is $\mathcal{G}_{\eta-}$-measurable. As $\mathcal{G}_{\eta}$ is generated by the set of all the $Y_{\eta}$ for all $\mathbb{G}$ optional processes $Y$, this proves the result.

\subsection{No cure period}

If $\delta=0$, then the expression for $\xi$ in $(5.1)$ reduces to

$$
\xi=\mathbb{1}_{\left\{\tau=\tau_{c}\right\}}\left(1-R_{c}\right)\left(P_{\tau}+\Delta_{\tau}-C_{\tau}^{c}\right)^{+}-\mathbb{1}_{\left\{\tau=\tau_{b}\right\}}\left(1-R_{b}\right)\left(P_{\tau}+\Delta_{\tau}-C_{\tau}^{b}\right)^{-},
$$

where $\Delta_{\tau}=D_{\tau}-D_{\tau-}$. Moreover, for every process $U=P,\left(D-D_{-}\right), C^{c}$ and $C^{b}$, there exists by 6.2) a $\mathcal{P}(\mathbb{G}) \otimes \mathcal{E}$-measurable function $\widetilde{U}=\widetilde{U}_{t}^{e}$ such that $U_{\tau}=\widetilde{U}_{\tau}^{e}$ holds on the event $\left\{\tau=\tau_{e}\right\}$, for each $e \in E$. In the present case where $\delta=0$, the process $\Delta$ only matters through $\Delta_{\tau}$, which is equal to $\left(D_{\tau}-D_{\tau-}\right)$. Accordingly, to alleviate the notation, in the case of $U=\left(D-D_{-}\right)$, we rewrite $\widetilde{U}_{t}^{e}=\widetilde{\Delta}_{t}^{e}$. We also rewrite $\left(\widetilde{C^{c}}\right)^{e}$ as $\widetilde{C}^{c, e}$ and $\left(\widetilde{C^{b}}\right)^{e}$ as $\widetilde{C}^{b, e}$.

Consistent with 6.1), let us assume $\tau_{b}=\min _{e \in E_{b}} \tau_{e}$ and $\tau_{c}=\min _{e \in E_{c}} \tau_{e}$, where $E=E_{b} \cup E_{c}$ (not necessarily a disjoint union, as will be exploited in Sect.7).

We may then take in 6.4 (where $\bar{\xi}=\xi$ when $\delta=0$ )

$$
\begin{aligned}
\widetilde{\xi}_{t}^{e}= & \mathbb{1}_{e \in E_{c}}\left(1-R_{c}\right)\left(\widetilde{P}_{t}^{e}+\widetilde{\Delta}_{t}^{e}-\widetilde{C}_{t}^{c, e}\right)^{+} \\
& -\mathbb{1}_{\left\{e \in E_{b}\right\}}\left(1-R_{b}\right)\left(\widetilde{P}_{t}^{e}+\widetilde{\Delta}_{t}^{e}-\widetilde{C}_{t}^{b, e}\right)^{-},
\end{aligned}
$$

so that by 6.3 , we have on $\llbracket 0, \bar{\tau} \rrbracket$ :

$$
\begin{aligned}
c d v a_{t}= & \left(1-R_{c}\right) \sum_{e \in E_{c}} \gamma_{t}^{e}\left(\widetilde{P}_{t}^{e}+\widetilde{\Delta}_{t}^{e}-\widetilde{C}_{t}^{c, e}\right)^{+} \\
& -\left(1-R_{b}\right) \sum_{e \in E_{b}} \gamma_{t}^{e}\left(\widetilde{P}_{t}^{e}+\widetilde{\Delta}_{t}^{e}-\widetilde{C}_{t}^{b, e}\right)^{-},
\end{aligned}
$$

where the two terms are interpreted as a CVA and a DVA coefficient. 
Hence, in the no cure period $\delta=0$ case, 5.3 is rewritten, on $\llbracket 0, \bar{\tau} \rrbracket$, as

$$
\begin{aligned}
\widehat{f}_{t}(\vartheta)+\left(r_{t}+\gamma_{t}\right) \vartheta= & \underbrace{\left(1-R_{c}\right) \sum_{e \in E_{c}} \gamma_{t}^{e}\left(\widetilde{P}_{t}^{e}+\widetilde{\Delta}_{t}^{e}-\widetilde{C}_{t}^{c, e}\right)^{+}}_{c v a_{t}} \\
& -\underbrace{\left(1-R_{b}\right) \sum_{e \in E_{b}} \gamma_{t}^{e}\left(\widetilde{P}_{t}^{e}+\widetilde{\Delta}_{t}^{e}-\widetilde{C}_{t}^{b, e}\right)^{-}}_{d v a_{t}} \\
& +\underbrace{c_{t} C_{t}+\tilde{\lambda}_{t}\left(P_{t}-C_{t}-\vartheta\right)^{+}-\lambda_{t}\left(P_{t}-C_{t}-\vartheta\right)^{-}}_{f v a_{t}(\vartheta)}
\end{aligned}
$$

where we set $\widetilde{\lambda}_{t}=\bar{\lambda}_{t}-\Lambda \sum_{e \in E_{b}} \gamma_{t}^{e}$.

\section{Part II}

\section{Credit derivatives TVA modelling}

In the second part of the paper, we apply the above approach to counterparty risk on credit derivatives traded between the bank and the counterparty respectively labeled as -1 and 0 , i.e., for $\tau_{b}=\tau_{-1}$ and $\tau_{c}=\tau_{0}$, and referencing names in $N=\{-1,0,1, \ldots, n\}$, for some nonnegative integer $n$.

Specifically, we will consider CDO tranches with upfront payment and CDS contracts corresponding to the respective dividend processes of the form, for $0 \leq t \leq T$ :

$$
\begin{aligned}
D_{t} & =\left(\left((1-R) \sum_{j \in N} \mathbb{1}_{\left\{t \geq \tau_{j}\right\}}-(n+2) a\right)^{+} \wedge(n+2)(b-a)\right) \text { Nom } \\
D_{t}^{i} & =\left(\left(1-R_{i}\right) \mathbb{1}_{\left\{t \geq \tau_{i}\right\}}-\left(t \wedge \tau_{i}\right) S_{i}\right) \text { Nom }_{i},
\end{aligned}
$$

for CDO tranches attachment and detachment points $a \leq b$ in [0, 100]\%, CDS contractual spreads $S_{i}$, recoveries $R$ and $R_{i}$ and nominals Nom and $\mathrm{Nom}_{i}$.

Our study will be conducted in the dynamic Marshall-Olkin (DMO) copula or common-shock model of Bielecki et al. [3]). As we shall see in detail, in the ensuing DMO model of TVA on credit derivatives, gap risk is already present for $\delta=0$, instantaneously realized in the joint default dividend $\Delta_{\tau}=D_{\tau}-D_{\tau-}$ (cf. Figure 9.2 and the surrounding comments below). Hence in this model it is enough to consider $\delta=0$, which we assume in the sequel.

By contrast, for counterparty risk on other kinds of derivatives, once a position is fully collateralized in terms of variation margins, then, for $\delta=0$, the continuously variation-margined TVA is equal to zero and gap risk only appears with a positive cure period $\delta>0$. The reader is referred to Armenti and Crépey [1, Sect. 8.5], where an impact in $\sqrt{\delta}$ is observed in the context of counterparty risk on interest rate derivatives.

Here is a summary list of notations introduced in the second part of the paper. 
$\mathcal{Y}$ Family of "shocks", i.e., subsets $Y \subseteq N=\{-1,0,1, \ldots, n\}$ of names likely to default together.

$\eta_{Y}, \gamma^{Y}, B^{Y}$ Shock time $\eta_{Y}$ with intensity $\gamma^{Y}$ deterministic or driven by an independent Brownian motion $B^{Y}$.

$\mathcal{Y}_{b}=\{Y \in \mathcal{Y} ;-1 \in Y\}, \mathcal{Y}_{c}=\{Y \in \mathcal{Y} ; 0 \in Y\}, \mathcal{Y}_{\bullet}=\mathcal{Y}_{b} \cup \mathcal{Y}_{c}, \mathcal{Y}_{\circ}=\mathcal{Y} \backslash \mathcal{Y}_{\bullet}$. The subsets of the shocks triggering the default of the bank, of the counterparty, of at least one of them, of none of them.

$\tau_{i}=\min _{\{Y \in \mathcal{Y} ; i \in Y\}} \eta_{Y}, \tau_{b}=\tau_{-1}=\min _{Y \in \mathcal{Y}_{b}} \eta_{Y}, \quad \tau_{c}=\tau_{0}=\min _{Y \in \mathcal{Y}_{c}} \eta_{Y}$ Default time of name $i$ in the DMO model, of the bank (i.e., name -1), of the counterparty (i.e., name 0).

$\gamma_{t}^{Y}, H_{t}^{Y}=\mathbb{1}_{\left\{\eta_{Y} \leq t\right\}}$ DMO Markov primitives.

$X_{t}=\left(\boldsymbol{\Gamma}_{t}, \mathbf{H}_{t}\right)$ where $\boldsymbol{\Gamma}=\left(\gamma^{Y}\right)_{Y \in \mathcal{Y}}, \mathbf{H}=\left(H^{Y}\right)_{Y \in \mathcal{Y}}$ Full DMO model Markov factor process.

$\widetilde{X}_{t}=\left(\boldsymbol{\Gamma}_{t}, \widetilde{\mathbf{H}}_{t}\right)$ where $\boldsymbol{\Gamma}=\left(\gamma^{Y}\right)_{Y \in \mathcal{Y}}, \widetilde{\mathbf{H}}=\left(\mathbb{1}_{Y \in \mathcal{Y}_{\circ}} H^{Y}\right)_{Y \in \mathcal{Y}}$. Reduced DMO model factor process.

Any function involving discrete arguments is viewed as continuous with respect to these, in reference to the discrete topology. When a process $f_{t}$ can be represented in terms of a function of some factor process $X$, we write $f\left(t, X_{t}\right)$, i.e., the function is denoted by the same letter as the process.

\section{Common-shock TVA model}

\subsection{Dynamic Marshall-Olkin model of default times}

We define a family $\mathcal{Y}$ of "shocks", i.e., subsets $Y$ of $N$, usually consisting of the singletons $\{-1\},\{0\},\{1\}, \ldots,\{n\}$ and of a few "common shocks" representing simultaneous defaults. The shock intensities are given in the form of extended CIR processes as, for every $Y \in \mathcal{Y}$,

$$
d \gamma_{t}^{Y}=a\left(b_{Y}(t)-\gamma_{t}^{Y}\right) d t+c \sqrt{\gamma_{t}^{Y}} d B_{t}^{Y},
$$

for nonnegative constants $a$ and $c$, continuous functions $b_{Y}(t)$ and independent Brownian motions $B^{Y}$ in their joint completed filtration $\mathbb{B}=\left(\mathcal{B}_{t}\right)_{t \geq 0}$, under the risk-neutral measure $\mathbb{Q}$. In fact, one could use in (7.1) any independent and square integrable Markov processes $\gamma^{Y} \geq 0$ provided $\mathbb{E} e^{-\int_{0}^{t} \gamma_{s}^{Y} d s}$ can be computed efficiently, which is required for calibration purposes. The case of deterministic intensities $\gamma_{t}^{Y}=b_{Y}(t)$ is treated in a similar fashion.

The shock random times and their indicator processes are defined by

$$
\eta_{Y}=\inf \left\{t>0 ; \int_{0}^{t} \gamma_{s}^{Y} d s>\epsilon_{Y}\right\} \text { and } H_{t}^{Y}=\mathbb{1}_{\left\{\eta_{Y} \leq t\right\}}, \quad Y \in \mathcal{Y},
$$

where the $\epsilon_{Y}$ are i.i.d. standard exponential random variables. In particular, the random times $\eta_{Y}$ avoid each other. The full model filtration $\mathbb{G}$ is given as $\mathbb{B}$ progressively enlarged by the random times $\eta_{Y}, Y \in \mathcal{Y}$. Let $M^{Y}$ denote the compensated martingale $d M_{t}^{Y}=d H^{Y}-\left(1-H_{t}^{Y}\right) \gamma_{t}^{Y} d t, \quad Y \in \mathcal{Y}$. 
Theorem 7.1 The dynamic Marshall-Olkin or common-shock model is a recursively immersed model of default times. For $t \geq 0$, we have

$$
\mathcal{G}_{t}=\mathcal{B}_{t} \vee \bigvee_{Y \in \mathcal{Y}}\left(\sigma\left(\eta_{Y} \wedge t\right) \vee \sigma\left(\left\{\eta_{Y}>t\right\}\right)\right) .
$$

The $B^{Y}$ and the $M^{Y}, Y \in \mathcal{Y}$, have the $(\mathbb{G}, \mathbb{Q})$ martingale representation property.

Proof We prove the martingale representation property and $(7.3)$ by induction as follows. We write $\mathbb{G}=\mathbb{G}^{\mathcal{Y}}$. If $\mathcal{Y}$ is a singleton (case of a Cox time in view of $(7.2)$ ), then the immersion of $\mathbb{B}$ into $\mathbb{G}^{\mathcal{Y}}$ implies the results, by the optional splitting formula (6.5) for (7.3) and by Jeanblanc and Song [22, Theorem 6.4] for the martingale representation property. Moreover, if $\mathcal{Z}$ is obtained by addition of a new $Z \subseteq N$ to $\mathcal{Y}$, then the independence of the $\epsilon_{Y}$ implies that $\eta_{Z}$ is a Cox time with intensity with respect to $\mathbb{G}^{\mathcal{Y}}$, hence immersion of $\mathbb{G}^{\mathcal{Y}}$ into $\mathbb{G}^{\mathcal{Z}}$ follows (this is the recursively immersed feature stated in the lemma) and the results for $\mathbb{G}^{\mathcal{Z}}$ are implied likewise from those, if assumed, for $\mathbb{G}^{\mathcal{Y}}$.

See also Crépey et al. [10, Chapters 8-10] regarding the Markov copula properties of the full DMO model factor process $X=(\boldsymbol{\Gamma}, \mathbf{H})$, where $\boldsymbol{\Gamma}=\left(\gamma^{Y}\right)_{Y \in \mathcal{Y}}$, $\mathbf{H}=\left(H^{Y}\right)_{Y \in \mathcal{Y}}$.

The empirical study reported in Crépey et al. [10, Sect. 8.4.3] shows that the DMO model is efficiently calibratable to CDS and CDO market data, including at the peak of the 2007-2008 credit crisis.

\subsection{TVA model}

Defining $\tau_{i}=\min _{\{Y \in \mathcal{Y} ; i \in Y\}} \eta_{Y}, i \in N$, a DMO model can be used as a marked stopping times credit derivatives TVA model, with

$E_{b}=\mathcal{Y}_{b}:=\{Y \in \mathcal{Y} ;-1 \in Y\}, \quad E_{c}=\mathcal{Y}_{c}:=\{Y \in \mathcal{Y} ; 0 \in Y\}, E=\mathcal{Y}_{\bullet}:=\mathcal{Y}_{b} \cup \mathcal{Y}_{c}$

Hence

$$
\tau_{b}=\tau_{-1}=\min _{Y \in \mathcal{Y}_{b}} \eta_{Y}, \quad \tau_{c}=\tau_{0}=\min _{Y \in \mathcal{Y}_{c}} \eta_{Y}
$$

and $\tau=\min _{Y \in \mathcal{Y}_{\bullet}} \eta_{Y}$, with intensity

$$
\gamma=J_{-} \sum_{Y \in \mathcal{Y}} \gamma^{Y}
$$

of $\tau$. The results of Crépey et al. [10, Corollary 8.3.1] show that for any portfolio of vanilla credit derivatives on names in $N$, i.e., $\mathrm{CDO}$ tranches and CDS contracts as of 6.8), the (trade-additive) risk-free price $P$ of the portfolio is of the form

$$
P_{t}=P\left(t, \boldsymbol{\Gamma}_{t}, \mathbf{H}_{t}\right),
$$

for some explicit continuous function $P$. 
We assume that for every process $U=P,\left(D-D_{-}\right), C^{c}$ and $C^{b}$, there exists a continuous function $\widetilde{U}=\widetilde{U}_{Y}(t, \boldsymbol{\gamma}, \mathbf{k})$ such that

$$
U_{\tau}=\widetilde{U}_{Y}\left(\tau, \boldsymbol{\Gamma}_{\tau}, \mathbf{H}_{\tau-}\right) \text { holds on }\left\{\tau=\eta_{Y}\right\}, \quad Y \in \mathcal{Y}_{\bullet} .
$$

To alleviate the notation we sometimes write $\widetilde{U}_{\tau}^{Y}$ instead of $\widetilde{U}_{Y}\left(\tau, \boldsymbol{\Gamma}_{\tau}, \mathbf{H}_{\tau-}\right)$ and we rewrite $\left(\widetilde{C^{c}}\right)^{Y}$ as $\widetilde{C}^{c, Y},\left(\widetilde{C^{c}}\right)_{Y}$ as $\widetilde{C}_{Y}^{c}$, etc.. Moreover in the case of $U=D-D_{-}, \widetilde{U}$ is rewritten as $\widetilde{\Delta}$.

In the case of vanilla credit derivatives on names in $N$, the condition (7.6) always holds regarding $U=P$, by $(7.5)$ and the following trivial identity:

$$
P\left(\tau, \boldsymbol{\Gamma}_{\tau}, \mathbf{H}_{\tau}\right)=P\left(\tau, \boldsymbol{\Gamma}_{\tau}, \mathbf{H}_{\tau-}^{Y}\right) \text { on }\left\{\tau=\eta_{Y}\right\},
$$

where $\mathbf{k}^{Y}$ denotes the vector obtained from a vector $\mathbf{k} \in\{0,1\}^{\mathcal{Y}}$ by replacing the component with index $Y$ by 1 . In view of (6.8), the condition 7.6 also holds for $U=D-D_{-}$. As will be illustrated numerically in Figure 9.2 the corresponding $\widetilde{U}^{Y}=\widetilde{\Delta}^{Y}$ convey the gap risk effect in the DMO model. The conditions 7.6 on $U=C^{c}$ and $C^{b}$ may be satisfied or not depending on the margin scheme.

In view of (6.6), the coefficient $\widetilde{\xi}$ (in the present no cure period case where $\delta=0$ ) is given as

$$
\begin{aligned}
\widetilde{\xi}_{t}^{Y}= & \mathbb{1}_{Y \in \mathcal{Y}_{c}}\left(1-R_{c}\right)\left(\widetilde{P}_{t}^{Y}+\widetilde{\Delta}_{t}^{Y}-\widetilde{C}_{t}^{c, Y}\right)^{+} \\
& -\mathbb{1}_{Y \in \mathcal{Y}_{b}}\left(1-R_{b}\right)\left(\widetilde{P}_{t}^{Y}+\widetilde{\Delta}_{t}^{Y}-\widetilde{C}_{t}^{b, Y}\right)^{-}, \quad Y \in \mathcal{Y}_{\bullet} .
\end{aligned}
$$

The coefficient $\widehat{f_{t}}(\vartheta)$ in 6.7 is given, on $\llbracket 0, \bar{\tau} \rrbracket$, by

$$
\begin{aligned}
\widehat{f_{t}}(\vartheta)+\left(r_{t}+\gamma_{t}\right) \vartheta= & \left(1-R_{c}\right) \sum_{Y \in \mathcal{Y}_{c}} \gamma_{t}^{Y}\left(\widetilde{P}_{t}^{Y}+\widetilde{\Delta}_{t}^{Y}-\widetilde{C}_{t}^{c, Y}\right)^{+} \\
& -\left(1-R_{b}\right) \sum_{Y \in \mathcal{Y}_{b}} \gamma_{t}^{Y}\left(\widetilde{P}_{t}^{Y}+\widetilde{\Delta}_{t}^{Y}-\widetilde{C}_{t}^{b, Y}\right)^{-} \\
& +c_{t} C_{t}+\widetilde{\lambda}_{t}\left(P_{t}-C_{t}-\vartheta\right)^{+}-\lambda_{t}\left(P_{t}-C_{t}-\vartheta\right)^{-},
\end{aligned}
$$

where $\tilde{\lambda}_{t}=\bar{\lambda}_{t}-\Lambda \sum_{Y \in \mathcal{Y}_{b}} \gamma_{t}^{Y}$.

Let $\mathcal{Y}_{\circ}=\mathcal{Y} \backslash \mathcal{Y}_{\bullet}$ and let $\widetilde{X}_{t}=\left(\boldsymbol{\Gamma}_{t}, \widetilde{\mathbf{H}}_{t}\right)$, where $\widetilde{\mathbf{H}}=\left(\mathbb{1}_{Y \in \mathcal{Y}_{\circ}} H^{Y}\right)_{Y \in \mathcal{Y}}$. We assume that the processes $r, c, \lambda, \bar{\lambda}, P$ and $C$ are given before $\tau$ as continuous functions of $\left(t, \widetilde{X}_{t}\right)$. Regarding $P$, the identity $(7.5)$ shows that this property always holds in the case of vanilla credit derivatives on names in $N$. Note that, in view of the second identity in (7.4), this property is also verified by the process $\gamma$.

Theorem 7.2 The condition $(C)$ holds, for:

(DMO.1) A reference filtration $\mathbb{F}=\left(\mathcal{F}_{t}\right)$ in $(C .1)$ given as $\mathbb{B}$ progressively enlarged by the $\eta_{Y}, Y \in \mathcal{Y}_{\circ}$, which satisfies

$$
\mathcal{F}_{t}=\mathcal{B}_{t} \vee \bigvee_{Y \in \mathcal{Y}_{\circ}}\left(\sigma\left(\eta_{Y} \wedge t\right) \vee \sigma\left(\left\{\eta_{Y}>t\right\}\right)\right), t \geq 0
$$


(DMO.2) The probability measure $\mathbb{P}=\mathbb{Q}$ in (C.2), where the $B^{Y}, Y \in \mathcal{Y}$, and the $M^{Y}, Y \in \mathcal{Y}_{\circ}$, have the $(\mathbb{F}, \mathbb{P}=\mathbb{Q})$ martingale representation property;

(DMO.3) A Markov specification $\widetilde{f}_{t}(\vartheta)=\widetilde{f}\left(t, \widetilde{X}_{t}, \vartheta\right)$ of $(C .3)$, for the $(\mathbb{F}, \mathbb{P}=\mathbb{Q})$ jump diffusion $\widetilde{X}_{t}=\left(\boldsymbol{\Gamma}_{t}, \widetilde{\mathbf{H}}_{t}\right)$ and the function $\widetilde{f}=\widetilde{f}(t, \widetilde{x}, \vartheta)$ given, for any $\widetilde{x}=(t, \boldsymbol{\gamma}, \widetilde{\mathbf{k}})$ with $\boldsymbol{\gamma}=\left(\gamma_{Y}\right)_{Y \in \mathcal{Y}} \in \mathbb{R}_{+}^{\mathcal{Y}}$ and $\widetilde{\mathbf{k}}=\left(k_{Y}\right)_{Y \in \mathcal{Y}} \in\{0,1\}^{\mathcal{Y}}$ such that $k_{Y}=0$ if $Y \in \mathcal{Y}_{\bullet}$, by:

$$
\begin{aligned}
& \widetilde{f}(t, \widetilde{x}, \vartheta)+(r(t, \widetilde{x})+\gamma(t, \widetilde{x})) \vartheta \\
& =\left(1-R_{c}\right) \sum_{Y \in \mathcal{Y}_{c}} \gamma_{Y}\left(\widetilde{P}_{Y}+\widetilde{\Delta}_{Y}-\widetilde{C}_{Y}^{c}\right)^{+}(t, \widetilde{x}) \\
& \quad-\left(1-R_{b}\right) \sum_{Y \in \mathcal{Y}_{b}} \gamma_{Y}\left(\widetilde{P}_{Y}+\widetilde{\Delta}_{Y}-\widetilde{C}_{Y}^{b}\right)^{-}(t, \widetilde{x}) \\
& \quad+\left(c C+\widetilde{\lambda}(P-\vartheta-C)^{+}-\lambda(P-\vartheta-C)^{-}\right)(t, \widetilde{x}),
\end{aligned}
$$

where $\gamma(t, \tilde{x})=\sum_{Y \in \mathcal{Y}_{\bullet}} \gamma_{Y}, \tilde{\lambda}=\bar{\lambda}-\Lambda \sum_{Y \in \mathcal{Y}_{b}} \gamma_{Y}$.

In addition, $(\mathbb{F}, \mathbb{P}=\mathbb{Q})$ local martingales do not jump at $\tau$, $\tau$ avoids $\mathbb{F}$ stopping times, the condition $(B)$ is satisfied and the Azéma supermartingale $S$ of $\tau$ is given, for $t \in[0, T]$, by

$$
S_{t}=e^{-\sum_{Y \in \mathcal{Y}} \int_{0}^{t} \gamma_{s}^{Y} d s}>0 .
$$

In particular, the DMO model is a marked default times setup satisfying (6.2), where the full and reduced BSDEs are equivalent.

Proof (DMO.1) and (DMO.2) can be addressed as the analogous statements in Theorem 7.1. (DMO.3) follows by inspection from (7.7).

By virtue of (7.8) in (DMO.1) and by the independence assumptions in the construction of the DMO model, we have

$$
\begin{aligned}
S_{t} & =\mathbb{Q}\left(\tau>t \mid \mathcal{F}_{t}\right)=\mathbb{Q}\left(\tau>t \mid \mathcal{B}_{t} \vee \bigvee_{Y \in \mathcal{Y}_{\circ}}\left(\sigma\left(\eta_{Y} \wedge t\right) \vee \sigma\left(\left\{\eta_{Y}>t\right\}\right)\right)\right) \\
& =\mathbb{Q}\left(\eta_{Y}>t, Y \in \mathcal{Y}_{\bullet} \mid \mathcal{B}_{t} \vee \bigvee_{Y \in \mathcal{Y}_{\circ}}\left(\sigma\left(\eta_{Y} \wedge t\right) \vee \sigma\left(\left\{\eta_{Y}>t\right\}\right)\right)\right) \\
& =\mathbb{Q}\left(\eta_{Y}>t, Y \in \mathcal{Y}_{\bullet} \mid \mathcal{B}_{t}\right)=\prod_{Y \in \mathcal{Y}_{\bullet}} \mathbb{Q}\left(\eta_{Y}>t \mid \mathcal{B}_{t}\right)=e^{-\sum_{Y \in \mathcal{Y}_{\bullet}} \int_{0}^{t} \gamma_{s}^{Y} d s} .
\end{aligned}
$$

(DMO.1-2-3) obviously imply (C.1) and (C.3). Each ( $\mathbb{F}, \mathbb{Q})$ martingale in (DMO.2), stopped at $(\tau-)$ or, equivalently by avoidance between the times $\eta_{Y}$, stopped at $\tau$, is a $(\mathbb{G}, \mathbb{Q})$ local martingale. Hence, (DMO.2) implies (C.2) via the $(\mathbb{F}, \mathbb{Q})$ martingale representation property that is included in (DMO.2). This proves that the condition (C) holds with $\mathbb{F}$ as of $(7.8), \mathbb{P}=\mathbb{Q}$ and $\widetilde{f}_{t}=\widetilde{f}\left(t, \widetilde{X}_{t}\right)$ (cf. (7.9)). The $(\mathbb{F}, \mathbb{Q})$ martingale representation property in (DMO.2) also shows that $(\mathbb{F}, \mathbb{Q})$ local martingales don't jump at $\tau$.

By He et al. [20, Theorem 5.27 1)] applied to the indicator process $\mathbb{1}_{\{\tau=\nu\}}$, where $v$ is an arbitrary $\mathbb{F}$ predictable stopping time, we have $\mathbb{Q}(\tau=v)=0$ as soon as the 
$(\mathbb{F}, \mathbb{Q})$ drift of $S$ is continuous, as it is in the DMO model in view of the formula $(7.10)$ for $S$. Besides, by the $(\mathbb{F}, \mathbb{Q})$ martingale representation property in (DMO.2), the $(\mathbb{F}, \mathbb{Q})$ compensated martingale of the default indicator process of an $\mathbb{F}$ totally inaccessible stopping time $v$ only jumps at the $\eta_{Y}, Y \in \mathcal{Y}_{\circ}$. Hence it cannot jump at $\tau$, i.e., $\mathbb{Q}(\tau=v)=0$. We conclude that $\tau$ avoids all $\mathbb{F}$ stopping times.

To check the condition (B), by the monotone class theorem, we only need consider the elementary $\mathbb{G}$ predictable processes of the form $U=v f\left(\left(\eta_{Y} \wedge s\right)_{\left.Y \in \mathcal{Y}_{\bullet}\right)}\right) \mathbb{1}_{(s, t]}$, for an $\mathcal{F}_{s}$-measurable random variable $v$ and a Borel function $f$. Since $U \mathbb{1}_{\rrbracket 0, \tau \rrbracket}=v f(s, \ldots, s) \mathbb{1}_{(s, t]} \mathbb{1}_{\rrbracket 0, \tau \rrbracket}$, we may take $U^{\prime}=v f(s, \ldots, s) \mathbb{1}_{(s, t]}$ in the condition (B).

The statements in the last line of the theorem follow from the other results by Lemma 6.3 and in view of the explanations given in the last paragraph of Sect. 5

Corollary 7.3 In the DMO model, we have $\gamma^{\prime}=\gamma(\cdot, \tilde{X})=.\sum_{Y \in \mathcal{Y} .} \gamma^{Y} \in \widetilde{\mathcal{H}}_{2}$. Assuming all the other conditions in Theorem 5.1 and without cure period, so for $\delta=0$ and $\widetilde{f}=\widetilde{f}(t, \boldsymbol{\gamma}, \widetilde{\mathbf{k}}, \vartheta)$ as of $(7.9)$, the corresponding reduced TVA BSDE (4.3) admits a unique square integrable solution $\widetilde{\Theta}_{t}=\widetilde{\Theta}\left(t, \widetilde{X}_{t}\right)$, where the function $\widetilde{\Theta}(t, \widetilde{x})$ is a continuous viscosity solution to the corresponding semilinear PIDE A solution $\Theta$ to the full TVA BSDE 3.5 is obtained by setting $\Theta=\widetilde{\Theta}$ on $\llbracket 0, \bar{\tau} \llbracket$ and

$$
\Theta_{\bar{\tau}}=\mathbb{1}_{\{\tau<T\}}\left(\widetilde{\xi}_{\tau}^{l}-\left(P_{\tau-}-C_{\tau-}-\widetilde{\Theta}_{\tau-}\right)^{+} \mathbb{1}_{\{-1 \in l\}} \Lambda\right),
$$

where $\imath \in \mathcal{Y}_{\bullet}$ is the identity of the shock triggering the first default of a party. The $(\mathbb{G}, \mathbb{Q})$ local martingale component $\mu$ of $\Theta$ satisfies, for $t \in \llbracket 0, \bar{\tau} \rrbracket$ :

$$
\begin{aligned}
d \mu_{t}= & d \widetilde{\mu}_{t}-\left(\widetilde{\xi}_{\tau}^{l}-\widetilde{\Theta}_{\tau-}-\left(P_{\tau-}-C_{\tau-}-\widetilde{\Theta}_{\tau-}\right)^{+} \mathbb{1}_{\{-1 \in l\}} \Lambda\right) d J_{t} \\
& -\left(\sum_{Y \in \mathcal{Y}_{\bullet}}\left(\widetilde{\xi}_{t}^{Y}-\widetilde{\Theta}_{t}\right) \gamma_{t}^{Y}-\left(P_{t}-C_{t}-\widetilde{\Theta}_{t}\right)^{+} \Lambda \sum_{Y \in \mathcal{Y}_{b}} \gamma_{t}^{Y}\right) d t .
\end{aligned}
$$

Proof In view of Theorem 7.2 Theorems 4.2 and 5.1 apply. They directly yield most of the stated results. Since the well-posedness result for the reduced TVA BSDE in Theorem 5.1 includes the BSDE comparison property and the usual a priori bound and error BSDE estimates, the representation of the solution $\widetilde{\Theta}$. to the reduced DMO TVA BSDE in terms of a continuous viscosity solution to the corresponding semilinear PIDE (with continuous coefficients) follows from standard arguments (see e.g. Delong [15] or Crépey [8, Chapter 13]).

\section{Conditions of numerical experiments}

\subsection{FT numerical scheme}

Due to risky funding, the TVA equations are Lipschitz BSDEs. In the case of credit derivatives, they are also very high-dimensional. Hence, all numerical schemes relying on dynamic programming, such as purely backward deterministic PIDE schemes, but

\footnotetext{
${ }^{1}$ Not written as not used in the paper.
} 
also forward/backward simulation/regression BSDE schemes, are ruled out by the curse of dimensionality. For $n$ greater than a few units, the only feasible DMO TVA numerical schemes are purely forward simulation schemes. In this paper we proceed by Monte Carlo estimation of successive orders of the linear BSDE expansion of Fujii and Takahashi [17], dubbed "FT scheme" henceforth.

The FT scheme is based on an expansion of the solution to a BSDE as a series of solutions to linear BSDEs, where the next BSDE in the series uses the solution to the previous one as input data. Consider, for $\epsilon>0$ :

$$
\begin{aligned}
& \widetilde{\Theta}_{T}^{\epsilon}=0 \text { and } d \widetilde{\mu}_{t}^{\epsilon}:=d \widetilde{\Theta}_{t}^{\epsilon}-\epsilon \widetilde{f}\left(t, \widetilde{X}_{t}, \widetilde{\Theta}_{t}^{\epsilon}\right) d t \text { is an }(\mathbb{F}, \mathbb{P}) \text { local martingale on }[0, T] \\
& \begin{aligned}
\widetilde{\Theta}_{t}^{\epsilon}=\widetilde{\Theta}_{t}^{(0)}+\epsilon \widetilde{\Theta}_{t}^{(1)}+\epsilon^{2} \widetilde{\Theta}_{t}^{(2)}+\epsilon^{3} \widetilde{\Theta}_{t}^{(3)}+\cdots \\
\widetilde{f}\left(t, \widetilde{X}_{t}, \widetilde{\Theta}_{t}^{\epsilon}\right)=\widetilde{f}\left(t, \widetilde{X}_{t}, \widetilde{\Theta}_{t}^{0}\right) \\
\quad+\left(\epsilon \widetilde{\Theta}^{(1)}+\epsilon^{2} \widetilde{\Theta}^{(2)}+\epsilon^{3} \widetilde{\Theta}^{(3)}+\cdots\right) \partial_{\vartheta} \widetilde{f}\left(t, \widetilde{X}_{t}, \widetilde{\Theta}_{t}^{(0)}\right)+\cdots
\end{aligned}
\end{aligned}
$$

Then, formally: $\widetilde{\Theta}=\widetilde{\Theta}^{\epsilon=1}=\widetilde{\Theta}^{(0)}+\widetilde{\Theta}^{(1)}+\widetilde{\Theta}^{(2)}+\widetilde{\Theta}^{(3)}+\cdots$. The convergence of this expansion is studied in a diffusive setup in Takahashi and Yamada [27] and Gobet and Pagliarani [19]. Collecting all terms in $\epsilon^{i}, i=0, \ldots, 3$, we obtain $\widetilde{\Theta}^{(0)}=0$ and

$$
\begin{aligned}
& \widetilde{\Theta}_{t}^{(1)}=\widetilde{\mathbb{E}}\left[\int_{t}^{T} \widetilde{f}\left(s, \widetilde{X}_{s}, \widetilde{\Theta}_{s}^{(0)}=0\right) d s \mid \mathcal{F}_{t}\right], \\
& \widetilde{\Theta}_{t}^{(2)}=\widetilde{\mathbb{E}}\left[\int_{t}^{T} \partial_{\vartheta} \widetilde{f}\left(s, \widetilde{X}_{s}, \widetilde{\Theta}_{s}^{(0)}=0\right) \widetilde{\Theta}_{s}^{(1)} d s \mid \mathcal{F}_{t}\right], \\
& \widetilde{\Theta}_{t}^{(3)}=\widetilde{\mathbb{E}}\left[\int_{t}^{T} \partial_{\vartheta} \widetilde{f}\left(s, \widetilde{X}_{s}, \widetilde{\Theta}_{s}^{(0)}=0\right) \widetilde{\Theta}_{s}^{(2)} d s \mid \mathcal{F}_{t}\right] .
\end{aligned}
$$

The first two lines correspond to the identities (2.19) and (2.22) in Fujii and Takahashi [17, arXiv version]. Compared with the third line, the complete third order term comprises another component based on $\partial_{\vartheta^{2}}^{2} \widetilde{f}$. In our case, $\partial_{\vartheta^{2}}^{2} \widetilde{f}$ involves a Dirac measure via the terms $\left(P_{t}-C_{t}-\vartheta\right)^{ \pm}$in $f v a_{t}(\vartheta)$ (cf. (5.3)), so that we truncate the expansion to the term $\widetilde{\Theta}_{t}^{(3)}$ as above (see Gobet and Pagliarani [19] for more about this).

Moreover, we use the interacting particles implementation of these formulas provided in Fujii and Takahashi [18]. Namely, we randomize, based on independent exponential draws $\epsilon_{j}$ with parameters $\mu_{j}$, each time integral that intervenes in 8.1) either explicitly or implicitly through the terms $\widetilde{\Theta}_{s}^{(1)}$ and $\widetilde{\Theta}_{s}^{(2)}$. We then obtain from (8.1) by repeated applications of the tower rule :

$$
\begin{aligned}
\widetilde{\Theta}_{0}^{(1)}= & \widetilde{\mathbb{E}}\left[\mathbb{1}_{\epsilon_{1}<T} \frac{e^{\mu_{1} \epsilon_{1}}}{\mu_{1}} \widetilde{f}\left(\epsilon_{1}, \widetilde{X}_{\epsilon_{1}}, 0\right)\right], \\
\widetilde{\Theta}_{0}^{(2)}= & \widetilde{\mathbb{E}}\left[\mathbb{1}_{\epsilon_{1}+\epsilon_{2}<T} \frac{e^{\mu_{1} \epsilon_{1}+\mu_{2} \epsilon_{2}}}{\mu_{1} \mu_{2}} \partial_{\vartheta} \widetilde{f}\left(\epsilon_{1}, \widetilde{X}_{\epsilon_{1}}, 0\right) \widetilde{f}\left(\epsilon_{1}+\epsilon_{2}, \widetilde{X}_{\epsilon_{1}+\epsilon_{2}}, 0\right)\right] \\
\widetilde{\Theta}_{0}^{(3)}= & \widetilde{\mathbb{E}}\left[\mathbb{1}_{\epsilon_{1}+\epsilon_{2}+\epsilon_{3}<T} \frac{e^{\mu_{1} \epsilon_{1}+\mu_{2} \epsilon_{2}+\mu_{3} \epsilon_{3}}}{\mu_{1} \mu_{2} \mu_{3}}\right. \\
& \left.\times \partial_{\vartheta} \widetilde{f}\left(\epsilon_{1}, \widetilde{X}_{\epsilon_{1}}, 0\right) \partial_{\vartheta} \widetilde{f}\left(\epsilon_{1}+\epsilon_{2}, \widetilde{X}_{\epsilon_{1}+\epsilon_{2}}, 0\right) \widetilde{f}\left(\epsilon_{1}+\epsilon_{2}+\epsilon_{3}, \widetilde{X}_{\epsilon_{1}+\epsilon_{2}+\epsilon_{3}}, 0\right)\right] .
\end{aligned}
$$




\subsection{Numerical data}

We set $\Lambda=0$, which allows validating the results of the FT scheme 8.2 by a Monte Carlo estimate based on the full BSDE (3.5). In fact, further setting $R_{b}=1$ and $c=\lambda=0$, we have the following linear approximation formula for the time- 0 value of the solution to the full TVA BSDE (in the present setup where $\delta=0$ ):

$$
\begin{aligned}
\Theta_{0} & \approx \mathbb{E}\left[\mathbb{1}_{\{\tau<T\}} \beta_{\tau} \xi+\int_{t}^{\bar{\tau}} \beta_{s} g_{s}\left(P_{s}\right) d s\right] \\
& =\mathbb{E}\left[\mathbb{1}_{\left\{\tau=\tau_{c}<T\right\}} \beta_{\tau}\left(1-R_{c}\right)\left(P_{\tau}+\Delta_{\tau}-C_{\tau}\right)^{+}+\int_{t}^{\bar{\tau}} \beta_{s} \bar{\lambda}_{s}\left(P_{s}-C_{s}\right)^{+} d s\right] .
\end{aligned}
$$

For $\bar{\lambda}=0$, equality holds in the first line and a Monte Carlo loop based on 8.3 yields an unbiased estimate for $\Theta_{0}=\widetilde{\Theta}_{0}$, alternative to a Monte Carlo estimate for $\widetilde{\Theta}_{0}^{(1)}+\widetilde{\Theta}_{0}^{(2)}+\widetilde{\Theta}_{0}^{(3)}$ as of 8.2 . For $\bar{\lambda} \neq 0,8.3$ is only a linear approximation to $\Theta_{0}$.

Unless stated otherwise, the following numerical values are used:

$$
\begin{aligned}
& r=0, \quad R_{c}=40 \%, \quad \delta=0, \quad V=I^{c} \\
& \bar{\lambda}=100 \mathrm{bp}=0.01, \quad \mu_{j}=\frac{2}{T}, \quad m=10^{4},
\end{aligned}
$$

where $m$ is the number of runs that are used in all the Monte Carlo estimates.

We consider a DMO model with constant shock intensities and $n=120$ credit names (unless stated otherwise). Note that the dependence between names is all in the common shocks in this model. The stochasticity of the intensities is not crucial for the gap risk feature that we want to investigate here. Using deterministic (constant in this case) intensities allows speeding up the simulations. We take individual shock intensities $\gamma^{\{i\}}=10^{-4} \times(100+i)$, which increases from $101 \mathrm{bp}$ to $220 \mathrm{bp}$ as $i$ increases from 1 to 120 . We consider four nested groups of joint defaults, respectively consisting of the riskiest $3 \%, 9 \%, 21 \%$ and $100 \%$ (i.e., all) names, with respective shock intensities of 20,10, 6.67 and $5 \mathrm{bp}$. The counterparty (resp. the bank) is taken as the eleventh (resp. tenth) safest name in the portfolio.

In this model, we consider three CDO tranches of maturity $T=2$ years with upfront payment: an equity tranche, a mezzanine tranche and a senior tranche, corresponding to the respective pairs $(a, b)$ in $(6.8)$ given as $(0 \%, 3 \%),(3 \%, 14 \%)$ and $(14 \%, 100 \%)$. The recovery $R$ and the nominal Nom of all the tranches are taken as $40 \%$ and 100 , respectively.

\section{Numerical results}

\subsection{Gap risk}

Figure 9.1 shows the TVA values obtained by FT schemes of order 1 to 3 , for different levels of nonlinearity (unsecured borrowing spread $\bar{\lambda}$ ). The values of the risk-free prices $P_{0}$ of the equity, mezzanine and senior tranche are 229.65, 5.68 and 2.99. In view of these, the TVA numbers of Figure 9.1 are very high, especially for the 
higher tranches, considerably greater for instance in relative terms than the interest rate derivatives TVA numbers obtained in Crépey et al. [11].
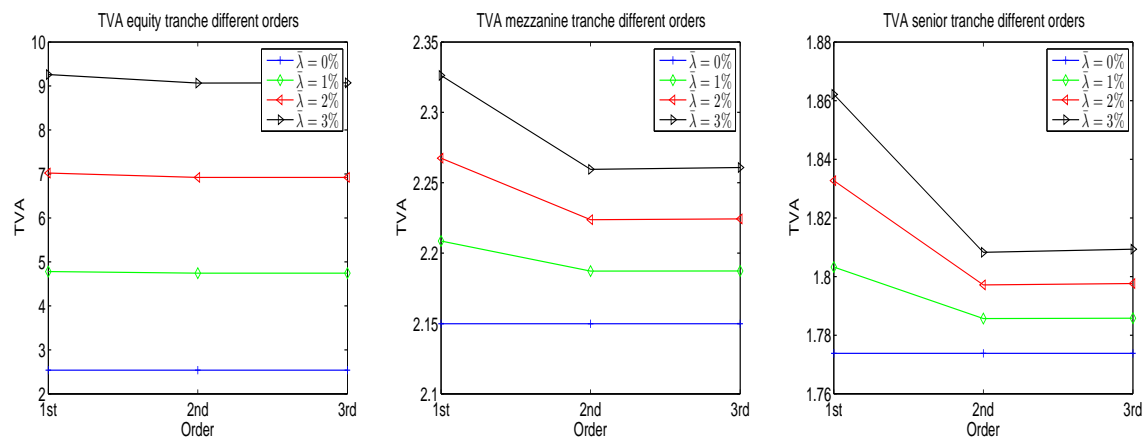

Fig. 9.1 TVA on CDO tranches with 120 underlying names computed by FT scheme of order 1 to 3 , for different levels of nonlinearity (unsecured borrowing spread $\bar{\lambda}$ ). Left: Equity tranche. Middle: Mezzanine tranche. Right: Senior Tranche.

Figure 9.2 shows the analog of Figure 9.1 using flawed simulations where we replace $\widetilde{P}_{t}^{e}+\Delta_{t}^{e}$ by $P_{t-}$ in the DMO coefficient $\widetilde{f}$ of $(7.9)$, removing the gap risk feature of the DMO model. Going from left to right and from top to bottom among these TVA curves, i.e., from the equity tranche to the senior tranche and from higher to smaller funding costs, the corresponding fake TVA numbers are $10 \%$ to one hundred times smaller than the "true" TVA numbers of Figure 9.1. We conclude that joint defaults are the main DMO CVA contributor, especially for higher tranches. Gap risk
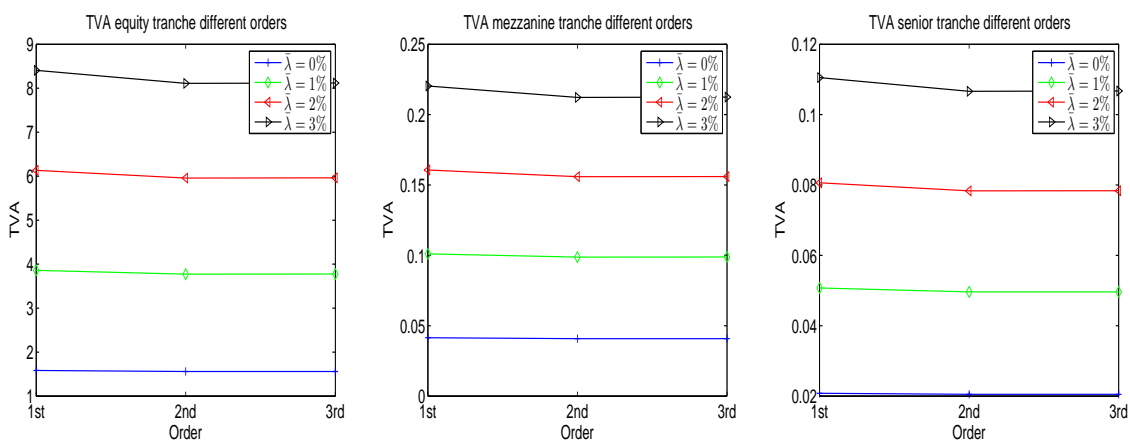

Fig. 9.2 Analog of Figure 9.1 in a fake DMO model without gap risk.

is already present for $\delta=0$ in the DMO model, instantaneously realized in the joint default dividend $\Delta_{\tau}=D_{\tau}-D_{\tau_{-}}$. This is also consistent with the findings in Bo and Capponi [6, Figure 1]. 


\subsection{Performance analysis of the FT scheme}

Depending on $\bar{\lambda}$ increasing from 0 to $300 \mathrm{bp}(\bar{\lambda}=0$ is a linear TVA case with higher order FT terms all equal to 0 ), the second (resp. third) FT term in (8.2) never exceeds in each case more than $5 \%$ of the first (resp. second) FT term in Figure 9.1. We conclude that the first FT term can be used as a first order linear estimate of the TVA, with a nonlinear correction given by the second FT term.

In Figure 9.3, which is computed for the mezzanine tranche, the left graph shows that the \% relative standard errors (\% rel. SE) of the different orders of the FT scheme do not explode with the dimension $n$. The middle graph, produced with $n=120$, shows that these errors do not explode with the level of nonlinearity $\bar{\lambda}$. Consistent with the fact that the successive FT terms are computed by standard Monte Carlo loops, the computation times are essentially linear in $n$, as visible in the right graph.
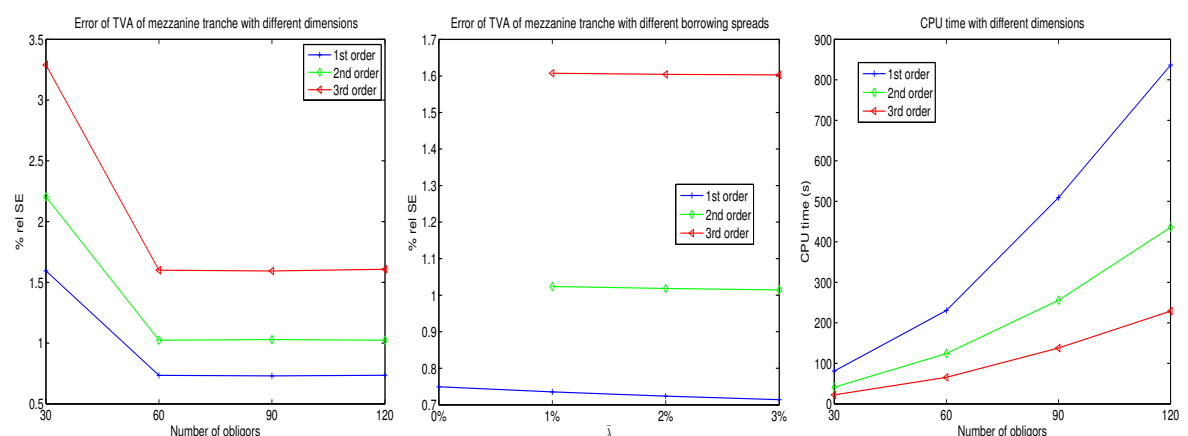

Fig. 9.3 Successive orders of the FT scheme for the mezzanine tranche of the middle panel of Figure 9.1 Left: The $\%$ relative standard errors do not explode with the number of names $(\bar{\lambda}=100 \mathrm{bp})$. Middle: The $\%$ relative standard errors do not explode with the level of nonlinearity represented by the unsecured borrowing spread $\bar{\lambda}(n=120)$. Right: The computation times are linear in the number of names $(\bar{\lambda}=100$ bp).

Table 9.1 compared with Figures 9.1 and 9.3 shows that on top of being biased (depending on $\bar{\lambda}$, equal to $100 \mathrm{bp}$ in Table 9.1), a Monte Carlo estimate based on the linear approximation formula 8.3 has a much larger variance, especially for higher tranches. In fact, for higher tranches, nonzero payoffs become quite rare events, so that exploiting the knowledge of the intensities in an FT scheme greatly improves the variance with respect to a Monte Carlo estimate based on (8.3). In addition, the

\begin{tabular}{|c||c|c|c||c||c|c|c|}
\hline Tranche & TVA & Rel. SE & $95 \%$ CI & Tranche & TVA & Rel. SE & $95 \%$ CI \\
\hline Eq. & 5.00 & $7.43 \%$ & {$[4.63,5.37]$} & Eq. & 4.94 & $2.40 \%$ & {$[4.82,5.06]$} \\
\hline Mezz. & 2.05 & $63.25 \%$ & {$[0.75,3.34]$} & Mezz. & 2.14 & $19.60 \%$ & {$[1.72,2.55]$} \\
\hline Sen. & 1.67 & $64.59 \%$ & {$[0.59,2.75]$} & Sen. & 1.74 & $20.02 \%$ & {$[1.39,2.09]$} \\
\hline
\end{tabular}

Table 9.1 Linearized DMO TVA on CDO tranches computed by Monte Carlo based on 8.3$)(\bar{\lambda}=100 \mathrm{bp})$. Left: $m=10^{4}$. Right: $m=10^{5}$. 
simulations for 8.3 take considerably more time, due to the discretisation of the time integral (or, if the latter was computed by randomization, then this would increase the variance even further).

\subsection{Impact of the margins}

We now consider the continuous variation-margining case where $V_{\tau}=P_{\tau-}$, as opposed to $V=0$ above. The left-limit in time in $P_{\tau-}$ accounts for an "(at least) infinitesimal delay" in the implementation of the margin calls, which in practice are performed according to some discrete-time schedule.

Even in this continuous variation-margining case and for $\delta=0$, we have

$$
Q_{\tau^{\delta}}-C_{\tau}^{c}=\left(P_{\tau^{\delta}}-P_{\tau-}\right)+\Delta_{\tau^{\delta}}-I_{\tau}^{c}=\left(P_{\tau}-P_{\tau-}\right)+\Delta_{\tau}-I_{\tau}^{c},
$$

where we already saw the importance of the gap term $\Delta_{\tau}=D_{\tau}-D_{\tau-}$ (the term $P_{\tau}-P_{\tau-}$ matters little in the DMO model). Accordingly, observe from Table 9.2 that the ensuing TVA numbers are still important relatively to the corresponding values of $P_{0}$, especially for higher tranches (and again, the FT scheme performs much better than the biased Monte Carlo based on (8.3) ). By comparison with Table 9.1, we see that it is only for the equity tranche that the TVA is substantially reduced by the variation margins. The TVA of higher tranches, essentially due to the common shocks, cannot be mitigated by variation margining.

\begin{tabular}{|c||c|c|c||c||c|c|c|}
\hline Tranche & TVA & Rel. SE & 95\% CI & Tranche & TVA & Rel. err. & $95 \%$ CI \\
\hline Eq. & 0.99 & $5.02 \%$ & {$[0.96,0.99]$} & Eq. & 1.02 & $17.02 \%$ & {$[0.84,1.19]$} \\
\hline Mezz. & 2.12 & $4.94 \%$ & {$[2.09,2.15]$} & Mezz. & 1.95 & $66.51 \%$ & {$[0.65,3.24]$} \\
\hline Sen. & 1.76 & $4.94 \%$ & {$[1.74,1.79]$} & Sen. & 1.62 & $66.64 \%$ & {$[0.54,2.70]$} \\
\hline
\end{tabular}

Table 9.2 TVA computations in the continuous variation-margining case $V_{\tau}=P_{\tau-}\left(\bar{\lambda}=100 \mathrm{bp}, m=10^{4}\right)$. Left: FT scheme based on 8.2 . Right : Monte Carlo based on the formula 8.3 .

This motivates the need for initial margins $I^{c}$. But the left panel of Table 9.3 shows that the amount of initial margins $I^{c}$, assumed a constant proportional to $\Theta_{0}$, that is required to balance the DMO gap term $\Delta_{\tau}=D_{\tau}-D_{\tau-}$, is huge, especially for the higher tranches. This is consistent with the extreme tail event feature of the CVA on senior protection CDO tranches. As seen above, most of the CVA comes from the few joint default scenarios giving rise to the joint default dividend $\Delta_{\tau}=D_{\tau}-D_{\tau-}$. To compete with these, initial margins must be of the same level of magnitude, i.e., very large, and this at every point in time of every possible scenario, as DMO default times are totally inaccessible. Such levels of initial margins would represent a huge funding charge for the counterparty. About the difficulty of mitigating joint to default risk by collateralization, see also Bo and Capponi [6, last paragraph of Sect. 3.1]. 


\begin{tabular}{|c||c|c|c|c|c|}
\hline Tranche $/ I^{c}$ & $\Theta_{0}$ & $10 \Theta_{0}$ & $10^{2} \Theta_{0}$ & $10^{3} \Theta_{0}$ & $10^{4} \Theta_{0}$ \\
\hline \hline Eq. & 0.97 & 0.85 & 0.16 & 0.00 & 0.00 \\
\hline Mezz. & 2.12 & 2.11 & 1.99 & 0.87 & 0.00 \\
\hline Sen. & 1.77 & 1.76 & 1.66 & 0.73 & 0.00 \\
\hline
\end{tabular}

Table 9.3 Values of $\Theta_{0}$ computed by FT scheme for different levels of initial margin $I^{c}$ posted by the counterparty $\left(V_{\tau}=P_{\tau-}\right)$.

\section{References}

1. Armenti, Y. and Crépey S. (2015). Central clearing valuation adjustment. Available online at http://arxiv.org/abs/1506.08595\#

2. Bichuch, M., Capponi A., and Sturm S. (2015). Arbitrage-free pricing of XVA Part I: Framework and explicit examples, followed by Part II: PDE representation and numerical analysis. Available online at http://arxiv.org/abs/1501.05893 and http://arxiv.org/abs/1502.06106

3. Bielecki, T. R., Cousin A., Crépey S., and Herbertsson A. (2014). Dynamic hedging of portfolio credit risk in a Markov copula model. Journal of Optimization Theory and Applications 161:90-102.

4. Bielecki, T. R., Jeanblanc M., and Rutkowski M. (2009). Credit Risk Modeling. Osaka University Press, Osaka University CSFI Lecture Notes Series 2.

5. Bielecki, T. R. and Rutkowski M. (2015). Valuation and hedging of contracts with funding costs and collateralization. SIAM Journal on Financial Mathematics 6:594-655.

6. Bo, L. and Capponi A. (2015). Counterparty risk for CDS: Default clustering effects. Journal of Banking and Finance 52:29-42.

7. Brigo, D. and Pallavicini A. (2014). Nonlinear consistent valuation of CCP cleared or CSA bilateral trades with initial margins under credit, funding and wrong-way risks. Journal of Financial Engineering 1, 1450001 (60 pages).

8. Crépey, S. (2013). Financial Modeling: A Backward Stochastic Differential Equations Perspective. Springer Finance Textbooks.

9. Crépey, S. (2015). Bilateral counterparty risk under funding constraints. Part II: CVA. Mathematical Finance 25:23-50.

10. Crépey, S., Bielecki T. R., and Brigo D. (2014). Counterparty Risk and Funding: A Tale of Two Puzzles. Chapman \& Hall/CRC Financial Mathematics Series.

11. Crépey, S., Gerboud R., Grbac Z., and Ngor N. (2013). Counterparty risk and funding: The four wings of the TVA. International Journal of Theoretical and Applied Finance 16(2), 1350006 (31 pages).

12. Crépey, S. and Song S. (2015a). BSDEs of counterparty risk. Stochastic Processes and their Applications 125:3023-3052.

13. Crépey, S. and Song S. (2015b). Invariance times. Available online at https://math.maths.univ-evry.fr/crepey

14. Cvitanic, J. and Karatzas I. (1993). Hedging contingent claims with constrained portfolios. Annals of Applied Probability 3:652-681.

15. Delong, L. (2013). Backward Stochastic Differential Equations with Jumps and their Actuarial and Financial Applications. Springer. 
16. El Karoui, N., Peng S., and Quenez M.-C. (1997). Backward stochastic differential equations in finance. Mathematical Finance 7:1-71.

17. Fujii, M. and Takahashi A. (2012). Analytical approximation for non-linear FBSDEs with perturbation scheme. International Journal of Theoretical and Applied Finance 15(5), 1250034(24).

18. Fujii, M. and Takahashi A. (2015). Perturbative expansion technique for nonlinear FBSDEs with interacting particle method. Asia-Pacific Financial Markets 22:283-304.

19. Gobet, E. and Pagliarani S. (2015). Analytical approximations of BSDEs with nonsmooth driver. SIAM Journal on Financial Mathematics 6:919-958.

20. He, S.-W., Wang J.-G., and Yan J.-A. (1992). Semimartingale Theory and Stochastic Calculus. CRC Press.

21. Hull, J. and White A. (2012). The FVA debate. Risk Magazine, July 83-85.

22. Jeanblanc, M. and Song S. (2013). Martingale representation theorem in progressively enlarged filtrations. Stochastic Processes and their Applications 125:42424271.

23. Korn, R. (1995). Contingent claim valuation in a market with different interest rates. Math. Meth. Oper. Res. 42:255-274.

24. Kruse, T. and Popier A. (2016). BSDEs with monotone generator driven by Brownian and Poisson noises in a general filtration. Stochastics: An International Journal of Probability and Stochastic Processes 88:491-539.

25. Song, S. (2014a). Local martingale deflators for asset processes stopped at a default time $s^{\mathfrak{t}}$ or right before $s^{\mathfrak{t}-}$. Working paper. Available online at http://arxiv.org/abs/1405.4474

26. Song, S. (2014b). Optional splitting formula in a progressively enlarged filtration. ESAIM Probability and Statistics 18:829-853.

27. Takahashi, A. and Yamada T. (2015). An asymptotic expansion of forwardbackward SDEs with a perturbed driver. International Journal of Financial Engineering 2(2): 1550020 . 